\title{
ONE DIMENSIONAL T.T.T STRUCTURES
}

\author{
DANIEL LOWENGRUB
}

\section{INTRODUCTION}

The notion of a first order topological structure was introduced by Pillay [1] as a generalization of the notion of an o-minimal structure. The idea is to provide a general framework in which model theory can be used to analyze a topological structure whose topology isn't necessarily induced by a definable order. In the o-minimal case, the topology is generated from a basis where each basis set can be defined by substituting the variables $y_{1}$ and $y_{2}$ by suitable parameters in the following formula

$$
\phi\left(x, y_{1}, y_{2}\right)=y_{1}<x<y_{2}
$$

A first order topological structure generalizes this to the case where $\phi$ is some arbitrary formula with more than one variable.

Pillay also introduced the notion of topologically totally transcendental (t.t.t) structures which have the additional property that any definable set has a finite number of connected components. For example, by definition o-minimal structures are t.t.t.

In the previously mentioned paper, Pillay proved that one dimensional t.t.t structures have some characteristics in common with o-minimal structures such as the exchange property. Furthermore, he showed that if the topology of a one dimensional t.t.t structure is induced by a definable dense linear ordering then the structure is o-minimal.

In this paper we'll focus on $\omega$-saturated one dimensional t.t.t structures and prove that under a few additional topological assumptions, such structures are composed of o-minimal components in a relatively simple manner.

Our main result which will be proved in section 4 will be to show that if we assume that removing any point from the structure splits it into at least two connected components, then the structure must be a one dimensional simplex of a finite number of o-minimal structures:

Theorem 1. Let $M$ be a 1-dimensional connected $\omega$-saturated t.t.t structure such that for each point $x \in M, M \backslash\{x\}$ has at least two definably connected components. Then there exists a finite set $X \subset M$ such that each of the finite number of connected components of $M \backslash X$ are o-minimal.

In section 5 we'll analyze the case where removing a point doesn't necessarily split the structure, and will find two alternative topological properties which guarantee that the structure is locally o-minimal. This is done by showing that basis sets which are small enough can be split by removing a point.

Theorem 2. Let $M$ be a 1-dimensional $\omega$-saturated t.t.t structure such that one of the following holds: 
(1) There exist a definable continuous function $F: M^{2} \rightarrow M$ and a point $a \in M$ such that for each $x \in M, F(x, x)=a$ and $F(x, \cdot)$ is injective.

(2) For every basis set $U,|b d(U)|=2$.

Then for all but a finite number of points, for every point $x \in M$ there's a basis set $U$ containing $x$ such that $U$ is o-minimal.

An immediate corollary of part 1 of theorem 2 is that if an $\omega$-saturated one dimensional t.t.t structure admits a topological group structure then it is locally o-minimal.

Towards the end of the section we'll prove a version of the monotonicity theorem for locally o-minimal structures. This shows that locally o-minimal structures share many characteristics with standard o-minimal structures.

Theorem 1 illustrates that the defining characteristic of o-minimal structures within the general setting of $\omega$-saturated one dimensional t.t.t structures isn't existence of the order itself, but rather the ability to disconnect the structure by removing a point.

Theorem 2 shows that even in the case where an $\omega$-saturated one dimensional t.t.t structure isn't o-minimal, it will at least be o-minimal on a local scale provided that it has a rudimentary internal structure.

An important step in proving the theorems above will be to show that the relation $a \sim_{x} b$, which says that $a$ and $b$ are in the same connected component of $M \backslash\{x\}$, is definable.

Proposition. Let $M$ be a 1-dimensional $\omega$-saturated t.t.t structure such that for each point $x \in M, M \backslash\{x\}$ has more than one connected component. Then the relation $a \sim_{x} b \subset M^{3}$ is definable.

In section 3 we'll prove that that the number of connected components in a definable family is uniformly bounded.

Proposition. Let $(M, \phi)$ be a 1-dimensional $\omega$-saturated t.t.t structure and

$$
\alpha\left(x, y_{1}, \ldots, y_{l}\right) \in L
$$

. Then there exists a constant $C \in \mathbb{N}$ such that for every l-tuple $c_{1}, \ldots, c_{l} \in M$, $\alpha\left(c_{1}, \ldots, c_{l}\right)$ has less than $C$ connected components.

This in turn will allow us to prove that elementary extensions of such structures are t.t.t as well.

\section{Preliminaries}

In this section we review some of the notions, definitions and results from Pillay [1] which will be used heavily throughout the following sections.

Definition 3. Let $M$ be a two sorted $L$ structure with sorts $M_{t}$ and $M_{b}$ and let $\phi\left(x, y_{1}, \ldots, y_{k}\right)$ be an $L$ formula such that $\left\{\phi^{M_{t}}(x, \bar{a}) \mid \bar{a} \in M_{b}^{k}\right\}$ is a basis for a topology on $M_{t}$. Then the pair $(M, \phi)$ will be called a first order topological structure. When we talk about the topology of $M_{t}$ we mean the one generated by the basis described above.

Remark. In Pillay's paper, first order topological structures were defined on a onesorted structure where each element can be both a parameter for a basis set, and a point in the topological space. However, in practice this double meaning isn't 
needed, so we're using the two sorted definition both for clarity and in order to slightly strengthen some of the theorems.

In addition, we consider the following condition on a first order topological structure $M$ :

(A) Every definable set $X \subset M_{t}$ is a boolean combination of definable open subsets.

In this paper we assume that $M_{t}$ is Hausdorff and $(M, \phi)$ is a first order topological structure satisfying (A).

The following topological result is also helpful in this context and was proved by Robinson [2, 4.2].

Lemma 4. Let $V$ be a topological space, and $W \subset V$ a non-empty subset. Let $A \subset V$ be a boolean combination of open subsets of $V$ and let $B=V \backslash A$. Then either $W \cap A$ or $W \cap B$ has an interior with respect to the induced topology on $W$.

Definition 5. Let $M$ be a first order topological structure satisfying (A) and let $X \subset M_{t}$ be a closed definable subset of $M_{t}$. The ordinal valued $D_{M}(X)$ is defined by:

(1) If $X \neq \emptyset$ then $D_{M}(X) \geq 0$.

(2) If $\delta$ is a limit ordinal and $D_{M}(X) \geq \alpha$ for all $\alpha<\delta$ then $D_{M}(X) \geq \delta$.

(3) If there's a closed definable $Y \subset M_{t}$ such that $Y \subset X, Y$ has no interior in $X$ and $D_{M}(Y) \geq \alpha$ then $D_{M}(X) \geq \alpha+1$.

Remark. We'll write $D_{M}(X)=\alpha$ if $D_{M}(X) \geq \alpha$ and $D_{M}(X) \nsupseteq \alpha+1$. We'll write $D_{M}(X)=\infty$ if $D_{M}(X) \geq \alpha$ for all $\alpha$.

Definition 6. We say that $M$ has dimension if $D_{M}(X) \neq \infty$ for all closed definable subsets $X \subset M_{t}$.

In addition, we define the number of definable connected components for definable subsets of our topology:

Definition 7. Let $X \subset M_{t}$ be definable. Then $d_{M}(X)$ is the maximum number $d<\omega$ such that there are disjoint definable clopen sets $X_{1}, \ldots, X_{d} \subset X$ with $X=\cup_{i=1}^{d} X_{i}$, and $\infty$ if no such $d$ exists.

Remark. Throughout the paper, when we say "connected" we always mean "definably connected".

And now for the main definition:

Definition 8. We say that $M$ is topologically totally transcendental (t.t.t) if $M$ is a first order topological structure satisfying (A) with dimension such that for every definable set $X \subset M_{t}, d_{M}(X)<\infty$. We say that a theory $T$ is t.t.t is every model of $T$ is t.t.t.

The following lemma was proved by Pillay $[1,6.6]$ and plays a key role in most of the proofs in this paper.

Lemma 9. Let $M$ be a 1-dimensional t.t.t structure. Then:

(1) For any closed and definable $X \subset M_{t}, D(X)=0$ iff $X$ is finite.

(2) The set of isolated points of $M_{t}$ is finite. 
(3) For any definable $X \subset M_{t}$ there are pairwise disjoint definably connected definable open subsets $X_{1}, \ldots, X_{m} \subset M_{t}$ and a finite set $Y \subset M_{t}$ such that $X=\left(\cup_{i=1}^{m} X_{i}\right) \cup Y$.

(4) For any definable $X \subset M_{t}$, the set of boundary points of $X$ is finite.

Remark. One consequence of part 3 of lemma 9 which will be used many times below is that if a set $A \subset M_{t}$ is definable then the statement " $A$ is infinite" is expressible in first order logic as it's equivalent to the statement "A has no interior".

\section{Connected Components in Definable Families}

In this section we'll show that the number of connected components is uniformly bounded over a definable family. This is used to prove that in 1-dimensional $\omega$-saturated structures, the property of being t.t.t is preserved under elementary equivalence.

Lemma 10. Let $(M, \phi)$ be a 1-dimensional $\omega$-saturated t.t.t structure. Then there exists a number $K \in \mathbb{N}$ such that for each point $b \in M_{b}, \mid b d\left(\phi^{M_{t}}(b) \mid \leq K\right.$.

Proof. For each $b \in M_{b}, \mid b d\left(\phi^{M_{t}}(b) \mid\right.$ is finite. The lemma then follows from the fact that $M$ is $\omega$-saturated.

Lemma 11. Let $(M, \phi)$ be a definably connected 1-dimensional $\omega$-saturated t.t.t structure, $K \in \mathbb{N}$ a number such that for each point $b \in M_{b}$ we have $\mid b d\left(\phi^{M_{t}}(b) \mid \leq\right.$ $K$, and $X \subset M_{t}$ a definable subset such that $b d(X)=n$. Then $d_{M}(X) \leq n \cdot K$.

Proof. Let $N=d_{M}(X)$ and let $\left\{Y_{1}, \ldots, Y_{N}\right\}$ be pairwise disjoint clopen (in $X$ ) subsets of $X$ such that $X=\cup_{i=1}^{N} Y_{i}$. In addition, we denote the elements of $b d(X)$ by $b d(X)=\left\{a_{1}, \ldots, a_{n}\right\}$.

By the Hausdorffness of $M_{t}$, we can find basis sets $\left\{U_{1}, \ldots, U_{n}\right\}$ such that for all $1 \leq i \leq n$ :

(1) $a_{i} \in U_{i}$

(2) For all $1 \leq j \leq N$, if $Y_{j} \neq\left\{a_{i}\right\}$ then $Y_{j} \backslash U_{i} \neq \emptyset$.

Claim. For all $1 \leq j \leq N$, if $Y_{j}$ isn't a point then there exists an $1 \leq i \leq n$ such that $a_{i} \in \bar{Y}_{i}$ and $b d\left(U_{i}\right) \cap Y_{j} \neq \emptyset$.

Proof. Let $1 \leq j \leq N$ be chosen such that $Y_{j}$ isn't a point. Without loss of generality, $Y_{j} \neq X$ because otherwise $X$ would be connected and the lemma would be trivial. Since $M_{t}$ is definably connected, $b d\left(Y_{j}\right) \neq \emptyset$. In addition, $Y_{j}$ is clopen in $X$ so $b d\left(Y_{j}\right) \subset b d(X)$. Therefore, there exists some $1 \leq i \leq n$ such that $a_{i} \in \bar{Y}_{i}$.

We'll now see that $b d\left(U_{i}\right) \cap Y_{j} \neq \emptyset$.

Assume for contradiction that $b d\left(U_{i}\right) \cap Y_{j}=\emptyset$. Then both $U_{i} \cap Y_{j}$ and $U_{i}^{c} \cap Y_{j}$ are non-empty clopen subsets of $X$, which is a contradiction to the fact that $Y_{j}$ is a connected component.

This completes the claim.

Without loss of generality, let's choose an integer $L$ between 1 and $N$ such that $\left\{Y_{1}, \ldots, Y_{L}\right\}$ are points and $\left\{Y_{L+1}, \ldots, Y_{N}\right\}$ are not points. Furthermore, let's choose an integer $M$ between 1 and $n$ such that $\left\{a_{1}, \ldots, a_{M}\right\}$ are isolated and $\left\{a_{M+1}, \ldots, a_{n}\right\}$ are not. It's clear that $L \leq M$.

According to the claim, for each index $L+1 \leq j \leq N$ there exists an integer $1 \leq i \leq n$ and a point $y_{j}$ such that $y_{j} \in b d\left(U_{i}\right)$ and $a_{i} \in \bar{Y}_{j}$. We note that from 
the fact that $a_{i} \in \bar{Y}_{j}$, it follows that $a_{i}$ is not an isolated point. This gives us a mapping:

$$
\phi:\left\{Y_{L+1}, \ldots, Y_{N}\right\} \rightarrow \bigcup_{i=M+1}^{n} b d\left(U_{i}\right)
$$

Since $y_{k} \neq y_{l}$ for each $L+1 \leq k<l \leq N$, the map $\phi$ is injective. Furthermore,

$$
\left|\bigcup_{i=M+1}^{n} b d\left(U_{i}\right)\right| \leq(n-M) \cdot K \leq(n-L) \cdot K
$$

so by the injectivity of $\phi$ we get that $N-L \leq(n-L) \cdot K$. But $K \geq 1$ so $N \leq n \cdot K$.

Proposition 12. Let $(M, \phi)$ be a 1-dimensional $\omega$-saturated t.t.t structure and let $\alpha\left(x, y_{1}, \ldots, y_{l}\right) \in L$ be a formula. Then there exists a constant $C \in \mathbb{N}$ such that for every l-tuple $c_{1}, \ldots, c_{l} \in M, d_{M}\left(\alpha^{M_{t}}\left(c_{1}, \ldots, c_{l}\right)\right)<C$.

Proof. First of all, let $K \in \mathbb{N}$ a number such that for each point $b \in M_{b}$ we have $\mid b d\left(\phi^{M_{t}}(b) \mid \leq K\right.$. By lemma 10, for each $\bar{c} \in M^{l}$ there exists a number $n_{\bar{c}} \in \mathbb{N}$ such that $b d\left(\alpha^{M_{t}}(\bar{c})\right)<n_{c}$. Therefore, since $M$ is $\omega$-saturated, there exists some $n \in \mathbb{N}$ such that for each tuple $\bar{c} \in M^{l}, b d\left(\alpha^{M_{t}}(\bar{c})\right)<n$.

We'll show that we can choose $C$ to be $d_{M}\left(M_{t}\right) \cdot K \cdot n$. Let $m=d_{M}\left(M_{t}\right)$ and let $\left\{Y_{1}, \ldots, Y_{m}\right\}$ be pairwise disjoint definably connected subsets such that $M_{t}=$ $\cup_{i=1}^{m} Y_{i}$. By lemma 11, for each $1 \leq i \leq m$ and $\bar{c} \in M^{l}, d_{M}\left(\alpha^{M_{t}}(\bar{c}) \cap Y_{i}\right)<n \cdot K$. The proposition then follows immediately.

We'll now use this boundedness result in order to prove that a certain set of first order properties are necessary and sufficient for an $\omega$-saturated first order topological structure to be t.t.t.

Theorem 13. Let $(M, \phi)$ be an $\omega$-saturated 1-dimensional t.t.t structure. Then $M$ has the following properties:

(1) For every formula $\alpha\left(x, y_{1}, \ldots, y_{l}\right) \in L$, there exists some $C \in \mathbb{N}$ such that for every tuple $\bar{c} \in M^{l}$, there exist $C$ points $x_{1}, \ldots, x_{C}$ in $\alpha^{M_{t}}(\bar{c})$ such that $\alpha^{M_{t}}(\bar{c}) \backslash\left\{x_{1}, \ldots, x_{C}\right\}$ is open.

(2) For every formula $\alpha\left(x, y_{1}, \ldots, y_{l}\right) \in L$, there exists a constant $C \in \mathbb{N}$ such that for all $c_{1}, \ldots, c_{l} \in M, d_{M}\left(\alpha^{M_{t}}\left(c_{1}, \ldots, c_{l}\right)\right)<C$.

(3) For any pair of formulas $\alpha\left(x, y_{1}, \ldots, y_{s}\right)$ and $\beta\left(x, y_{1}, \ldots, y_{t}\right)$ in $L$, and for all $\bar{a} \in M^{s}$ and $\bar{b} \in M^{t}$, if $B=\beta^{M_{t}}(\bar{b}) \subset \alpha^{M_{t}}(\bar{a})=A$ is closed and non empty and doesn't have an interior in $A$, then $A$ has an interior in $M_{t}$.

Furthermore, if $(M, \phi)$ is a first order topological structure which satisfies these three properties and is Hausdorff, then $M$ is a 1-dimensional t.t.t structure.

Proof. First we'll see that the three properties are sufficient. Assume that $(M, \phi)$ is a first order topological structure such that $M_{t}$ is Hausdorff and has the three properties in the theorem.

By property 1 , every definable set $X$ is a boolean combination of open sets so $M$ has property (A). By property 2, every definable set has a finite number of definably connected components. Finally, by property $3, D(M)=1$.

Now we'll prove the first part of the theorem. Let $(M, \phi)$ be an $\omega$-saturated t.t.t structure. By the definition of t.t.t, $M_{t}$ is Hausdorff. We'll now prove that $M$ has each one of the required properties. 
(1) Let $\alpha\left(x, y_{1}, \ldots, y_{l}\right) \in L$. Since $M$ is t.t.t, for every $\bar{c} \in M^{l}$, there exist $C$ points $x_{1}, \ldots, x_{C}$ in $\alpha^{M_{t}}(\bar{c})$ such that $\alpha^{M_{t}}(\bar{c}) \backslash\left\{x_{1}, \ldots, x_{C}\right\}$ is open. Since $M$ is $\omega$-saturated, we can choose $C$ uniformly for all $\bar{c} \in M^{l}$.

(2) This property is essentially proposition 12

(3) This follows from the fact that $D(M)=1$.

Corollary 14. Let $\phi\left(x, y_{1}, \ldots, y_{k}\right)$ be a formula and let $(M, \phi)$ be a 1-dimensional t.t.t structure which is $\omega$-saturated. In addition, let $N$ be a model such that $N \equiv M$. Then $(N, \phi)$ is a 1-dimensional t.t.t structure.

Proof. This is immediate from the fact that all of the properties in theorem 13 can be expressed in first order logic.

\section{Structures With Splitting}

4.1. Introduction. Our main result in this section is that for any 1-dimensional $\omega$-saturated t.t.t structure, if removing any point splits the space into more that one connected component then there exists a finite set $X \subset M_{t}$ such that each connected component of $M_{t} \backslash X$ is o-minimal.

In order to prove this, we first obtain some intermediate results such as the fact that the equivalence relation $y \sim_{x} z$ specifying if $y$ and $z$ are in the same connected component of $M_{t} \backslash\{x\}$ is a definable relation in $M_{t}^{3}$. We also introduce a notion of "local flatness" which is used as a stepping stone between t.t.t structures and o-minimality.

For example, consider the structure $R_{\text {int }}=\langle\mathbb{R}, I(x, y, z)\rangle$ where $I(x, y, z)$ is true if $z$ lies on the interval between $x$ and $y$. In example 22 we'll show that $R_{\text {int }}$ has the property that removing any point splits the space into more that one connected component. We'll use this fact to show that the relation $y \sim_{x} z$ is indeed definable. In the end of this section we'll demonstrate how applying the construction of the order to $R_{\text {int }}$ gives the standard ordering on the reals.

In this section we're assuming that $M_{t}$ has no isolated points. This doesn't pose a problem because $M_{t}$ has at most a finite number of isolated points so we can remove them without affecting any of our results.

4.2. A Definable Relation. The following equivalence relation is useful for analyzing what happens when a point is removed from a structure.

Definition 15. Let $M$ be a 1-dimensional t.t.t structure. Let $x, a, b \in M_{t}$. Then $a \sim_{x} b$ will be a relation which is true iff $a$ and $b$ are in the same definable connected component of $M_{t} \backslash\{x\}$.

Remark. Note that by proposition 12, there exists an $N \in \mathbb{N}$ such that for each point $x \in M_{t}, \sim_{x}$ has less than $N$ equivalence classes.

Our first goal is to show that if for every $x \in M_{t}$ we have $d_{M}\left(M_{t} \backslash\{x\}\right) \geq 2$, then $\sim_{x} \subset M_{t}^{3}$ is definable.

We start by showing that for any $x$ such that $d_{M}\left(M_{t} \backslash\{x\}\right)>2, x \in \operatorname{acl}(\emptyset)$.

The following technical lemma will be used many times throughout the proof.

Intuitively, the lemma says that after removing two points, the space is divided into three distinct components. The part "in between" the points we removed and one additional side for each of the points. 
Lemma 16. Let $M$ be a 1-dimensional $\omega$-saturated t.t.t structure, $C \subset M_{t}$ an open connected definable subset, $a \neq b \in C$ and $2 \leq k, l \in \mathbb{N}$ such that $d_{M}(C \backslash\{a\})=k$ and $d_{M}(C \backslash\{b\})=l$. Let $A_{1}, \ldots, A_{k}$ and $B_{1}, \ldots, B_{l}$ be the connected components of $C \backslash\{a\}$ and $C \backslash\{b\}$ respectively such that $a \in B_{1}$ and $b \in A_{1}$. Then:

(1) $b d\left(\cup_{i=2}^{k} A_{i}\right)=\{a\}$

(2) $b d\left(\cup_{j=2}^{l=2} B_{j}\right)=\{b\}$

(3) $b d\left(A_{1} \cap B_{1}\right)=\{a, b\}$ and for every open set $U$ containing a or $b, U \cap\left(A_{1} \cap\right.$ $\left.B_{1}\right) \neq \emptyset$.

(4) The following union is disjoint:

$$
C=\left(\cup_{i=2}^{k} A_{i}\right) \cup\{a\} \cup\left(A_{1} \cap B_{1}\right) \cup\{b\} \cup\left(\cup_{j=2}^{l} B_{j}\right)
$$

Proof. First we'll prove 1.

Since $C$ is connected, $a \in \overline{A_{i}}$ for each $1 \leq i \leq k$. Therefore, since $a \notin A_{i}$ for each $2 \leq i \leq k, a \in b d\left(\cup_{i=2}^{k} A_{i}\right)$. In addition, $\cup_{i=2}^{k} A_{i}$ is open which means that $b d\left(\cup_{i=2}^{k} A_{i}\right) \subset\{a\} \cup A_{1}$. But $A_{1}$ is open as well and disjoint to $\cup_{i=2}^{k} A_{i}$ so $b d\left(\cup_{i=2}^{k} A_{i}\right)=\{a\}$.

Similarly, $b d\left(\cup_{j=2}^{l} B_{j}\right)=\{b\}$.

We'll now show that $\cup_{i=2}^{k} A_{i} \subset B_{1}$. In order to do that we first prove that $\left(\cup_{i=2}^{k} A_{i}\right) \cup\{a\}$ is connected in $C \backslash\{b\}$. Assume for contradiction that $X_{1}$ and $X_{2}$ form a clopen partition of $\left(\cup_{i=2}^{k} A_{i}\right) \cup\{a\}$ in $C \backslash\{b\}$. Without loss of generality, $a \in X_{1}$ which means that $a \notin \overline{X_{2}}$. Furthermore, $A_{1}$ is open and $b \in A_{1}$ which means that $b \notin \overline{X_{2}}$. Together this means that $X_{2} \subset\left(\cup_{i=2}^{k} A_{i}\right)$ and $b \notin b d\left(X_{2}\right)$. Therefore, $X_{2}$ is clopen in $C$ which is a contradiction to the fact that $C$ is connected.

Now, $B_{1}$ is the connected component of $C \backslash\{b\}$ containing $a$. So from the fact that $\left(\cup_{i=2}^{k} A_{i}\right) \cup\{a\}$ is connected in $C \backslash\{b\}$ it follows that $\cup_{i=2}^{k} A_{i} \subset B_{1}$.

We're now ready to prove 4 . It's immediate that

$$
\left(\cup_{i=2}^{k} A_{i}\right) \cap\left(A_{1} \cap B_{1}\right)=\left(\cup_{j=2}^{l} B_{j}\right) \cap\left(A_{1} \cap B_{1}\right)=\emptyset
$$

In addition, since $\left(\cup_{i=2}^{k} A_{i}\right) \subset B_{1}$ it follows that $\left(\cup_{j=2}^{l} B_{j}\right) \cap\left(\cup_{i=2}^{k} A_{i}\right)=\emptyset$. This shows that the union is disjoint so all that's left is to show that it's equal to $C$.

Let $c \in C$ be a point such that

$$
c \notin\left(\cup_{j=2}^{l} B_{j}\right) \cup\{a\} \cup\left(\cup_{i=2}^{k} A_{i}\right) \cup\{b\}
$$

Since $c \in C \backslash\{a\}$ and $c \notin\left(\cup_{i=2}^{k} A_{i}\right)$ it follows that $c \in A_{1}$. Similarly, $c \in B_{1}$. Therefore, $c \in A_{1} \cap B_{1}$.

We're now ready to prove 3 .

First of all, assume for contradiction that $A_{1} \cap B_{1}=\emptyset$. Then by parts 1,2 and 4 of the lemma, the sets $\left(\cup_{i=2}^{k} A_{i}\right) \cup\{a\}$ and $\{b\} \cup\left(\cup_{j=2}^{l} B_{j}\right)$ would form a clopen partition of $C$ which is a contradiction to the assumption that $C$ is connected.

We'll now show that $b d\left(A_{1} \cap B_{1}\right)=\{a, b\}$ On the one hand, $a \in \operatorname{int}\left(B_{1}\right)$ and $a \in \overline{A_{1}}$ so $a \in b d\left(A_{1} \cap B_{1}\right)$. Similarly, $b \in A_{1} \cap B_{1}$. On the other hand, $A_{1} \cap B_{1}$ is open so

$$
b d\left(A_{1} \cap B_{1}\right) \subset\left(\cup_{i=2}^{k} A_{i}\right) \cup\{a\} \cup\{b\} \cup\left(\cup_{j=2}^{l} B_{j}\right)
$$

But $\cup_{i=2}^{k} A_{i}$ and $\cup_{j=2}^{l} B_{j}$ are open well so $b d\left(A_{1} \cap B_{1}\right) \subset\{a\} \cup\{b\}$. Together we get that $b d\left(A_{1} \cap B_{1}\right)=\{a, b\}$.

Finally, let $U$ be an open set containing $a$ or $b$. Since $b d\left(A_{1} \cap B_{1}\right)=\{a, b\}$, it follows that $U \cap\left(A_{1} \cap B_{1}\right) \neq \emptyset$. 
Lemma 17. Let $M$ be a 1-dimensional $\omega$-saturated connected t.t.t structure. Let $D \subset M_{t}$ be an open definable subset, $E(x, a, b) \subset M_{t}^{3} a$ definable relation and $N \in \mathbb{N}$ such that:

(1) $N \geq 2$.

(2) For every $x \in D$ and $a, b \in M_{t}, a \sim_{x} b \Rightarrow E(x, a, b)$.

(3) For every $x \in D, E(x, a, b)$ is an equivalence relation with $N$ classes.

Then for each point $a \in D$, there exists a point $b \in D$ such that the definable set $X=\{x \in D \mid \neg E(x, a, b)\}$ is infinite.

Proof. Let $a \in D$. Without loss of generality, $D$ is connected. Otherwise, we'll look at the connected component containing $a$. We'll now show that there exists a point $b \in D$ such that for an infinite number of points $x \in D$ we have $\neg E(x, a, b)$. In order to do this, we'll inductively construct a sequence of points $\left(b_{1}, b_{2}, \ldots\right)$ in $D$ such that for each $n \in \mathbb{N}$ and each $1 \leq j<n, a \sim_{b_{n}} b_{j}$ and $\neg E\left(b_{j}, a, b_{n}\right)$.

For $n=1$, we can choose any $b_{1} \in D \backslash\{a\}$.

Let's assume that we constructed the sequence up to $b_{n}$. Let $X_{1}, \ldots, X_{c\left(b_{n}\right)}$ be the connected components of $M_{t} \backslash\left\{b_{n}\right\}$ such that $a \in X_{2}$. We choose $b_{n+1} \in D$ to be some point such that $\neg E\left(b_{n}, a, b_{n+1}\right)$. By our assumptions on $E(x, a, b), b_{n+1} \notin X_{2}$. So without loss of generality, in $b_{n+1} \in X_{1}$. Let $Y_{1}, \ldots, Y_{c\left(b_{n+1}\right)}$ be the connected components of $M \backslash\left\{b_{n+1}\right\}$ such that $b_{n} \in Y_{1}$. By lemma 16, for all $1<j \leq c\left(b_{n+1}\right)$, $Y_{j} \cap X_{2}=\emptyset$. By the inductive hypothesis, $b_{j} \in X_{2}$ for all $1 \leq j<n$. This means that for all $1 \leq j<n, b_{j} \in Y_{1}$. Similarly, $a \in Y_{1}$ and we already know that $b_{n} \in Y_{1}$. Together we've shown that $a \sim_{b_{n+1}} b_{j}$ for all $1 \leq j<n+1$.

We'll now show that for all $1 \leq j<n, \neg E\left(b_{j}, a, b_{n+1}\right)$. This will be enough because we already know that $\neg E\left(b_{n}, a, b_{n+1}\right)$.

Let $j$ be an index such that $1 \leq j<n$. Let $X_{1}, \ldots, X_{c\left(b_{j}\right)}$ be the connected components of $M_{t} \backslash\left\{b_{j}\right\}$ such that $a \in X_{2}$ and $b_{n} \in X_{1}$. In addition, let $Y_{1}, \ldots, Y_{c\left(b_{n}\right)}$ be the connected components of $M_{t} \backslash\left\{b_{n}\right\}$ such that $b_{j}, a \in Y_{1}$ and $b_{n+1} \in Y_{2}$. Since $b_{j} \in Y_{1}$ and $b_{n} \in X_{1}$, by lemma 16 it follows that $Y_{2} \subset X_{1}$ which means that $b_{n} \sim_{b_{j}} b_{n+1}$. By our assumptions on $E$ this implies $E\left(b_{j}, b_{n}, b_{n+1}\right)$. Therefore, as $\neg E\left(b_{j}, a, b_{n}\right)$, we can conclude that $\neg E\left(b_{j}, a, b_{n+1}\right)$.

Now, by the $\omega$-saturation, there exists a point $b \in D$ such that $\mid\{x \in D$ : $\neg E(x, a, b)\} \mid=\infty$.

Lemma 18. Let $M$ be a 1-dimensional $\omega$-saturated connected t.t.t structure. Let $D \subset M_{t}$ be an open definable subset, $E(x, a, b) \subset M_{t}^{3}$ a definable relation and $N \in \mathbb{N}$ such that:

(1) For every $x \in D$ and $a, b \in M_{t}, a \sim_{x} b \Rightarrow E(x, a, b)$.

(2) For every $x \in D, E(x, a, b)$ is an equivalence relation with $N$ classes.

Then $N \leq 2$.

Proof. Assume for contradiction that $N>2$. For ease of notion, we define $c(x)=$ $d_{M}\left(M_{t} \backslash\{x\}\right)$ for each $x \in M_{t}$. We note that for all $x \in D, c(x)>2$.

By lemma 17, there exist points $a, b \in M_{t}$ such that for an infinite number of points $x \in D, \neg E(x, a, b)$.

We denote the infinite set $\{x \in D: \neg E(x, a, b)\}$ by $X$.

Let $x, y \in X$, let $X_{1}, \ldots, X_{c(x)}$ be the connected components of $M_{t} \backslash\{x\}$ such that $a \in X_{1}$ and $b \in X_{2}$ and let $Y_{1}, \ldots, Y_{c(y)}$ be the connected components of $M_{t} \backslash\{y\}$ such that $a \in Y_{1}$ and $b \in Y_{2}$. 
First we note that for every $j$ such that $3 \leq j \leq c(x), y \notin X_{j}$. Because let $j$ be an index such that $3 \leq j \leq n(x)$, let $k$ be an index such that $x \in Y_{k}$ and assume for contradiction that $y \in X_{j}$. Then, since $y \in X_{j}$ and $x \in Y_{k}$, it follows from lemma 16] that $X_{1}, X_{2} \subset Y_{k}$ which means that $a, b \in Y_{k}$. However, this contradicts our assumption that $a \nsim_{y} b$.

In an analogous fashion, for each index $j$ such that $3 \leq j \leq c(y)$ we have $x \notin Y_{j}$.

Therefore, $x \in Y_{1} \cup Y_{2}$ and $y \in X_{1} \cup X_{2}$.

Since this is true for any pair of points $x, y \in X$, by lemma 16 we get that:

(1) for all $3 \leq i \leq c(x)$ and $3 \leq j \leq c(y), X_{i} \cap Y_{j}=\emptyset$

(2) for all $3 \leq i \leq c(x), X_{i} \cap X=\emptyset$.

From these two results we'll show that that $M_{t} \backslash X$ has an infinite number of definable connected components. First of all, by 2 it follows that for each point $x \in X$, the classes of $E(x, a, b)$ not containing $a$ and $b$ are definable sets which are contained and clopen in $M_{t} \backslash X$. Furthermore, by 1, all the sets obtained this way are disjoint.

But since $X$ is both infinite and definable, this is a contradiction to the fact that $M$ is t.t.t.

Now, let $p$ be some type in $S(\emptyset)$. We now show that there exist a $\emptyset$-definable relation $R_{p}(x, a, b) \subset M_{t}^{3}$ and an infinite $\emptyset$-definable set $D_{p} \subset M_{t}$ such that:

(1) For all elements $x \models p$ and points $a, b \in M_{t}, R_{p}(x, a, b) \Longleftrightarrow a \sim_{x} b$.

(2) For all elements $x \in D_{p}$ and points $a, b \in M_{t}, a \sim_{x} b \Rightarrow R_{p}(x, a, b)$.

(3) For all elements $x \in D_{p}, R_{p}(x, a, b) \subset M_{t}^{2}$ is an equivalence relation with $d_{M}\left(M_{t} \backslash\{y\}\right)$ equivalence classes where $y$ is some element realizing $p$.

(4) For every element $x$ that realizes $p, x \in D_{p}$.

We construct $R_{p}$ and $D_{p}$ in the following way. First, let $x$ be some realization of $p$ and define $N$ by $N=d_{M}\left(M_{t} \backslash\{x\}\right)$. Then there exist $\phi_{1}(x, \bar{y}), \ldots, \phi_{N}(x, \bar{y})$ such that:

$\left(^{*}\right)$ for some $\bar{y}, \phi_{1}^{M_{t}}[\bar{y}], \ldots, \phi_{N}^{M_{t}}[\bar{y}]$ partition $M_{t} \backslash\{x\}$ into $N$ disjoint clopen sets. Furthermore, for any other $\bar{z}$, if $\left(\phi_{1}^{M_{t}}[\bar{z}], \ldots, \phi_{N}^{M_{t}}[\bar{z}]\right)$ is a partition of $M_{t} \backslash\{x\}$ into disjoint clopen sets then it's the same partition as $\left(\phi_{1}^{M_{t}}[\bar{y}], \ldots, \phi_{N}^{M_{t}}[\bar{y}]\right)$.

Since this is a first order statement, $(*)$ holds for all $x \models p$.

Now, we define $D_{p}$ as the set of all the points $x \in M_{t}$ such that $(*)$ holds for $x$ with the formulas $\phi_{1}(x, \bar{y}), \ldots, \phi_{N}(x, \bar{y})$. We then define $R_{p}(x, a, b)$ as a relation which is true iff for one of the points $\bar{y}$ guaranteed by $\left(^{*}\right)$ for $x$, the sets $\phi_{1}^{M_{t}}[\bar{y}], \ldots, \phi_{N}^{M_{t}}[\bar{y}]$ partition $M_{t} \backslash\{x\}$ into $N$ disjoint clopen sets such that $a$ and $b$ are in the same section of the partition.

Proposition 19. Let $M$ be a 1-dimensional $\omega$-saturated connected t.t.t structure and let $x \in M_{t}$ be a point such that $d_{M}\left(M_{t} \backslash\{x\}\right)>2$. Then $D_{t p(x / \emptyset)}$ is finite and in particular, $x \in \operatorname{acl}(\emptyset)$.

Proof. Let $N=d_{M}\left(M_{t} \backslash\{x\}\right)$ and $p=t p(x / \emptyset)$. Since $N>2$, by applying lemma 18 with $D=\operatorname{int}\left(D_{p}\right)$ and $E=R_{p}$, $\operatorname{int}\left(D_{p}\right)$ is finite. Therefore, $D_{p}$ is finite.

We now look at what happens if $d_{M}\left(M_{t} \backslash\{x\}\right)=2$.

As before, let $p \in S(\emptyset)$ be a type such that for some element $x$ realizing $p$ we have $d_{M}\left(M_{t} \backslash\{x\}\right)=2$. We define $\tilde{D}_{p} \subset D_{p}$ as the set of points $x \in D_{p}$ such that there exist elements $a, b \in M_{t}$ and a basis set $U \subset M_{t}$ containing $x$ such that for all points $u \in U, \neg R_{p}(u, a, b)$. 
Proposition 20. Let $M$ be a 1-dimensional $\omega$-saturated connected t.t.t structure and let $p \in S_{1}(\emptyset)$ be a complete type in $M_{t}$. In addition, assume that for some (all) elements $x \models p, \sim_{x}$ has 2 equivalence classes. Then, for each point $x$ realizing $p$, one of the following hold:

(1) There exists a finite $\emptyset$-definable subset of $D_{p}$ containing $x$ and in particular, $x \in \operatorname{acl}(\emptyset)$.

(2) $\operatorname{int}\left(\tilde{D}_{p}\right)$ is a set containing $x$ such that for every point $y \in \operatorname{int}\left(\tilde{D}_{p}\right), d_{M}\left(M_{t} \backslash\{y\}\right)=$ 2 .

Proof. First of all, if $D_{p}$ is finite then the first case holds for all $x \models p$.

Let's assume that $D_{p}$ is infinite. Now, suppose that for each point $x$ realizing $p$ :

$\left(^{*}\right)$ for all $a, b \in M_{t}$ and for every basis set $U$ containing $x$, there exists a point $u \in U$ such that $R(u, a, b)$.

We define the set $C \subset D_{p}$ as the set of points in $D_{p}$ with property (*). $C$ is clearly $\emptyset$-definable. Furthermore, for all $x \models p, x \in C$. Assume for contradiction that $C$ is infinite. In that case, by lemma 17 there exist points $a, b \in M_{t}$ and a basis set $U \subset C$ such that for all $u \in U, \neg R(u, a, b)$. This is clearly a contradiction to $\left(^{*}\right)$. This means that $C$ is finite so again we're in the first case for every point $x \models p$.

Therefore, we can assume that for all elements $x$ realizing $p, x \in \tilde{D}_{p}$.

If $\tilde{D}_{p}$ is finite then again we're in the first case for every point $x \models p$.

We'll now see that if $\tilde{D}_{p}$ is infinite then for each point $y \in \operatorname{int}\left(\tilde{D}_{p}\right), d_{M}\left(M_{t} \backslash\{y\}\right) \leq$ 2. This will finish the proposition because we already know that for every point $x \in \tilde{D}_{p}, d_{M}\left(M_{t} \backslash\{x\}\right) \geq 2$. We also note that if $x \in \tilde{D}_{p} \backslash \operatorname{int}\left(\tilde{D}_{p}\right)$ then clearly we're in the first case as $\left|\tilde{D}_{p} \backslash \operatorname{int}\left(\tilde{D}_{p}\right)\right|<\infty$.

Let's assume for contradiction that $y \in \operatorname{int}\left(\tilde{D}_{p}\right)$ and $d_{M}\left(M_{t} \backslash\{y\}\right)>2$. Since $y \in \tilde{D}_{p}$, there exist points $a, b \in M_{t}$ and a basis set $U \subset \tilde{D}_{p}$ containing $y$ such that for all $u \in U, \neg R_{p}(u, a, b)$. Let $Y_{1}, Y_{2}, Y_{3}$ be three definable disjoint clopen sets partitioning $M_{t} \backslash\{y\}$ such that $a \in Y_{1}$ and $b \in Y_{2}$. Since $M_{t}$ is connected, there exists a $z$ such that $z \in Y_{3} \cap U$. Since $z \in D_{p}, k=d_{M}\left(M_{t} \backslash\{z\}\right) \geq 2$. Let $Z_{1}, \ldots, Z_{k}$ be definable pairwise disjoint clopen sets partitioning $M_{t} \backslash\{z\}$ such that $y \in Z_{1}$. Since $y \in Z_{1}$ and $z \in Y_{3}$, by lemma [16 it follows that $Y_{1} \cup Y_{2} \subset Z_{1}$ which means that $a, b \in Z_{1}$. However, this is a contradiction to the fact that $\neg R_{p}(z, a, b)$.

We now use the previous two propositions to show that if for each point $x \in M_{t}$ we have $d_{M}\left(M_{t} \backslash\{x\}\right)>1$, then the relation $a \sim_{x} b \subset M_{t}^{3}$ is $\emptyset$-definable.

Proposition 21. Let $M$ be a 1-dimensional connected $\omega$-saturated t.t.t structure such that for each point $x \in M_{t}, d_{M}\left(M_{t} \backslash\{x\}\right)>1$. Then the relation $a \sim_{x} b \subset M_{t}^{3}$ is definable.

Proof. We'll show that both $a \sim_{x} b$ and $a \nsim_{x} b$ are $\bigvee$-definable by formulas without parameters.

$a \nsim_{x} b$ is clearly $\bigvee$-definable by formulas without parameters because $a \nsim_{x} b$ iff there exist two open sets whose boundary is $\{x\}$ such that one contains $a$ and the other contains $b$. 
We'll now prove that $a \sim_{x} b$ is $\bigvee$-definable by formulas without parameters. This is done by showing that for each point $x \in M_{t}$, there exists a set $C_{x} \subset M_{t}^{3}$ which is definable without parameters such that:

(1) For all points $y, a, b \in M_{t},(y, a, b) \in C_{x} \Rightarrow a \sim_{y} b$

(2) $(x, a, b) \in C_{x} \Longleftrightarrow a \sim_{x} b$

Let's choose some $x \in M_{t}$ and define $p=t p(x / \emptyset)$.

If $d_{M}\left(M_{t} \backslash\{x\}\right)=N>2$ then, by proposition 19, $D_{p}$ is a finite $\emptyset$-definable set containing $x$. Furthermore, for every $y \in D_{p}, d_{M}\left(M_{t} \backslash\{x\}\right) \geq N$. Let's denote the points in $D_{p}$ by $D_{p}=\left\{y_{1}, \ldots, y_{k}\right\}$. Without loss of generality, there exists some $0 \leq l<k$ such that for all $1 \leq i \leq l, d_{M}\left(M_{t} \backslash\left\{y_{i}\right\}\right)>N$ and for all $l+1 \leq i \leq k$, $d_{M}\left(M_{t} \backslash\left\{y_{i}\right\}\right)=N$. It's easy to see that for each $1 \leq i \leq l, x \notin D_{t p\left(y_{i} / \emptyset\right)}$. Therefore, we can define:

$$
C_{x}=\left(\left(D_{p} \backslash \bigcup_{i=1}^{l} D_{t p\left(y_{i} / \emptyset\right)}\right) \times M_{t}^{2}\right) \cap R_{p}
$$

. Finally, let's assume that $d_{M}\left(M_{t} \backslash\{x\}\right)=2$.

If $D_{p}$ contains a finite $\emptyset$-definable set containing $x$, then we can define $C_{x}$ in the same way as in the previous case. Otherwise, by proposition $20, \operatorname{int}\left(\tilde{D}_{p}\right) \subset D_{p}$ is a set containing $x$ such that for all $y \in \operatorname{int}\left(\tilde{D}_{p}\right), d_{M}\left(M_{t} \backslash\{y\}\right)=2$. Therefore, we can define:

$$
C_{x}=\left(\operatorname{int}\left(\tilde{D}_{p}\right) \times M_{t}^{2}\right) \cap R_{p}
$$

. This finishes the proof of the proposition.

Example 22. Let's look at the structure $R_{\text {int }}=\langle\mathbb{R}, I(x, y, z)\rangle$ where $I(x, y, z)$ is true if $z$ lies on the interval between $x$ and $y$. In other words:

$$
I(x, y, z)=\left\{(x, y, z) \in \mathbb{R}^{3} \mid(x<z<y) \vee(y<z<x)\right\}
$$

The basis sets will be given by:

$$
\left\{I^{R_{\text {int }}}(a, b, z) \mid a, b \in \mathbb{R}\right\}
$$

Since $\langle\mathbb{R},<\rangle$ is an $\omega$-saturated one dimensional t.t.t structure, so is $R_{\text {int }}$. We'll now see that for every point $a \in \mathbb{R}, \mathbb{R} \backslash\{a\}$ has two definably connected components in $R_{\text {int }}$.

Let $a$ be some point in $\mathbb{R}$. Let $c$ and $b$ be two constants in $\mathbb{R}$ such that $c<a<b$. Then:

$$
\begin{aligned}
& x<a \Longleftrightarrow I(x, a, c) \vee I(c, a, x) \\
& a<x \Longleftrightarrow I(a, x, b) \vee I(a, b, x)
\end{aligned}
$$

this shows that $\mathbb{R} \backslash\{a\}$ has two definably connected components in $R_{\text {int }}$.

Therefore, by proposition 21, the relation $a \sim_{x} b$ is definable in $R_{\text {int }}$. Indeed:

$$
a \sim_{x} b \Longleftrightarrow \neg I(a, b, x)
$$


4.3. Local and Global Flatness. We'll now prove that, under the condition that removing any point creates at least two connected components, there exist a finite number of points such that after removing them, the remaining finite number of connected components are o-minimal. This is done by first showing that up to a finite number of points the structure is "locally o-minimal", and then showing that local o-minimality implies global o-minimality. In addition, the definability of the relation $a \sim_{x} b \subset M_{t}^{3}$ will play a crucial role.

We start by defining a notion of "local flatness" and then showing that locally flat points have a neighborhood which behaves similarly to an o-minimal one.

Definition 23. Let $M$ be t.t.t structure. We say that the point $x \in M_{t}$ is locally flat if there exist points $a, b \in M_{t}$ and a basis set $U$ such that for every point $u \in U$, $a \nsim_{u} b$. We say that a set $D$ is locally flat if all of it's points are locally flat.

We first show that in the type of structures which we're currently interested in, all but a finite number of points are locally flat.

Proposition 24. Let $M$ be a 1-dimensional $\omega$-saturated connected t.t.t structure such that for each point $x \in M_{t}, d_{M}\left(M_{t} \backslash\{x\}\right)>1$. Then for all but a finite number of points $x \in M_{t}, x$ is locally flat.

Proof. Let $X \subset M_{t}$ be the set of points $x \in M_{t}$ such that for all points $a, b \in M_{t}$ and for every basis set $U$, there exists a point $y \in U$ such that $a \sim_{y} b$. By proposition 21. $X$ is definable.

Assume for contradiction that $X$ is infinite. Then, by lemma 17, there exists a pair of points $a, b \in M_{t}$ such that the set $\tilde{X}=\left\{x \in X \mid a \nsim_{x} b\right\}$ is infinite. In addition, $\tilde{X}$ is definable so there exists a basis set $U$ which is contained in $\tilde{X} \subset X$. This is clearly a contradiction to the definition of $X$.

The next few propositions will show that points which are locally flat have a neighborhood on which we can define a linear order. This motivates the "flatness" in the definition.

Lemma 25. Let $M$ be a 1-dimensional $\omega$-saturated connected t.t.t structure such that for all $x \in M_{t}, d_{M}\left(M_{t} \backslash\{x\}\right)>1$. Let $x \in M_{t}$ be locally flat and let $U \subset M_{t}$ be an open connected definable set containing $x$. Then $U \backslash\{x\}$ has two connected components.

Proof. Since $d_{M}\left(M_{t} \backslash\{x\}\right)>1$ and $M_{t}$ is connected, it's enough to show that $U \backslash\{x\}$ has no more than two connected components.

Assume for contradiction that $U_{1}, \ldots, U_{k}$ are the connected components of $U \backslash\{x\}$ with $k>2$. In addition, let $a, b \in M_{t}$ be points and let $V \subset U$ be a basis set containing $x$ such that for all $v \in V, a \nsim_{v} b$.

Let the sets $X_{1}, X_{2} \subset M_{t} \backslash U$ be part of a clopen partition of $M_{t} \backslash U$ such that $a \in X_{1}$ and $b \in X_{2}$. By the connectedness of $M_{t}$, there exist points $b_{1}, b_{2} \in b d(U)$ such that $b_{1} \in X_{1}$ and $b_{2} \in X_{2}$. Without loss of generality, $b_{1} \in b d\left(U_{1}\right)$ and $b_{2} \in b d\left(U_{2}\right)$. Furthermore, the connectedness of $M_{t}$ implies that $U_{3} \cap V \neq \emptyset$.

Now, let $y$ be a point in $U_{3} \cap V$. By lemma 16, $U_{1}$ and $U_{2}$ are both subsets of the connected component of $U \backslash\{y\}$ which contains $x$. So since $\overline{U_{1}} \cap X_{1} \neq \emptyset$ and $\overline{U_{2}} \cap X_{2} \neq \emptyset$, both $X_{1}$ and $X_{2}$ are in the same connected component of $M_{t} \backslash\{y\}$. This means that $a \sim_{y} b$ which is a contradiction to the fact that $y \in V$.

One consequence of lemma 25 which will be used later is that if we remove all of the finite number of points which aren't locally flat then for each of the remaining 
connected components $C$, and for each point $x \in C, C \backslash\{x\}$ will have exactly two connected components. This will be used to show that $C$ is o-minimal.

Our next goal is to define an order on some neighborhood of each locally flat point. Let $x_{0} \in U$ be locally flat and let $U \subset M_{t}$ be a connected definable neighborhood of $x_{0}$. We define a relation $<_{x_{0}, U}$ on $U$ in the following way.

By lemma 25, $U \backslash\left\{x_{0}\right\}$ has two connected components which we'll denote by $V_{+}$ and $V_{-}$. Let $x$ and $y$ be points in $U$. We'll say that $x<_{x_{0}, U} y$ if one of the following hold:

$$
\begin{aligned}
& \text { - } x, y \in V_{+} \text {and } x_{0} \nsim_{x} y \\
& \text { - } x, y \in V_{-} \text {and } x_{0} \nsim_{y} x \\
& \text { - } y \in V_{+} \text {and } x \in V_{-} . \\
& \text {- } y=x_{0} \text { and } x \in V_{-} . \\
& \text {- } x=x_{0} \text { and } y \in V_{+} .
\end{aligned}
$$

Note that by proposition 21, $x<_{x_{0}, U} y$ is definable.

We now show that if $x_{0}$ is locally flat then there exists a neighborhood $x_{0} \in U$ such that $<_{x_{0}, U}$ defines a dense linear order on $U$.

Proposition 26. Let $M$ be a 1-dimensional connected $\omega$-saturated t.t.t structure such that for all $x \in M_{t}, d_{M}\left(M_{t} \backslash\{x\}\right)>1$. Let $D \subset M_{t}$ be a connected open definable subset such that for every point $x \in D, d_{M}(M \backslash\{x\})=2$. Let $x_{0} \in D$ be a locally flat point. Then there exists a connected open neighborhood $U \subset D$ of $x_{0}$ such that $<_{x_{0}, U}$ defines a dense linear order on $U$.

Proof. Since $x_{0}$ is locally flat, there exist points $a, b \in M_{t}$ and a basis set $V \subset D$ such that $x_{0} \subset V$ and for every point $y \in V$ we have $a \nsim_{y} b$.

We can assume that $V$ is connected because otherwise we can take the connected component containing $x_{0}$. In addition, let $V_{+}$and $V_{-}$be the two connected components of $V \backslash\left\{x_{0}\right\}$ (by lemma 25 there are exactly two) and let $C_{a}$ and $C_{b}$ be the connected components of $M_{t} \backslash\left\{x_{0}\right\}$ such that $a \in C_{a}$ and $b \in C_{b}$. In addition, without loss of generality $V_{+} \subset C_{a}$ and $V_{-} \subset C_{b}$. This follows from the fact that $C_{a} \cap V$ and $C_{b} \cap V$ partition $V$ into two clopen sets so by lemma 25, one must equal $V_{+}$and the other must equal $V_{-}$.

We'll now show that $<_{x_{0}, V}$ is a dense linear order on $V$.

Let $x, y$ and $z$ be points in $V_{+}$. In addition, let $X_{a}$ and $X_{b}$ be the connected components of $M_{t} \backslash\{x\}$ such that $a \in X_{a}$ and $b \in X_{b} . Y_{a}, Y_{b}, Z_{a}$ and $Z_{b}$ are defined analogously for $y$ and $z$.

Since $x, y \in V_{+} \subset C_{a}$, it follows from lemma [16 that $x_{0} \in X_{b}$ and $x_{0} \in Y_{b}$. Because if we assume for contradiction that $x_{0} \in X_{a}$ then by lemma 16 we get that $C_{b} \cap X_{b}=\emptyset$ which is a contradiction to the fact that $b \in C_{b} \cap X_{b}$. The proof that $x_{0} \in Y_{b}$ is identical.

(1) $x \nless_{x_{0}, V} y \Rightarrow y<_{x_{0}, V} x$ :

According to the assumption, $x_{0} \sim_{x} y$ which together with the fact that $x_{0} \in X_{b}$ means that $y \in X_{b}$. Now let's assume for contradiction that $x \in Y_{b}$. Since $y \in X_{b}$ we get from lemma 16 that $Y_{a} \cap X_{a}=\emptyset$ which is a contradiction since $a \in X_{a} \cap Y_{a}$. Therefore, $x \in Y_{a}$ which together with $x_{0} \in Y_{b}$ gives $y<x_{o, V} x$.

(2) $x<_{x_{0}, V} y \Rightarrow y \nless_{x_{0}, V} x$ : 
Since $x<_{x_{0}, V} y$ and $x_{0} \in X_{b}$, we get that $y \in X_{a}$. Now, we claim that $x \in Y_{b}$. For otherwise, if $x \in Y_{a}$ then we'd get from lemma 16 that $Y_{b} \cap X_{b}=\emptyset$ which is a contradiction.

Therefore, since $x_{0} \in Y_{b}$ as well, $y \nless_{x_{0}, V} x$.

(3) $x<_{x_{0}, V} y \wedge y<_{x_{0}, V} z \Rightarrow x<_{x_{0}, V} z$ :

According to the assumptions, $x_{0}, x \in Y_{b}, z \in Y_{a}, x_{0} \in X_{b}$ and $y \in X_{a}$. We have to prove that $z \in X_{a}$ as well. But again by lemma 16, $y \in X_{a} \wedge x \in$ $Y_{b} \Rightarrow Y_{a} \subset X_{a}$.

The proof of these claims is either trivial or identical when $x, y$ and $z$ are distributed differently among $V_{+}, V_{-}$and $\left\{x_{0}\right\}$.

This shows that $<_{x_{0}, V}$ is indeed a linear order. We'll now show that if $x<_{x_{0}, V} y$ then there exists a point $z \in V$ such that $x<_{x_{0}, V} z<_{x_{0}, V} y$. Again we'll assume that $x, y \in V_{+}$. As before, this means that $x \in Y_{b}$ and $y \in X_{a}$ which by lemma [16] implies that $\left(X_{a} \cap Y_{b}\right) \cap V \neq \emptyset$. Let $z$ be some point in $\left(X_{a} \cap Y_{b}\right) \cap V$. By the definition of $<_{x_{0}, V}$ and the fact that it's linear we get that $x<_{x_{0}, V} z<_{x_{0}, V} y$.

Before extending the order defined to connected components, we introduce the notion of an interval in a t.t.t structure and prove some useful properties.

Definition 27. Let $M$ be a 1-dimensional t.t.t structure and $x, y \in M_{t}$ such that $d_{M}\left(M_{t} \backslash\{x\}\right)=d_{M}\left(M_{t} \backslash\{y\}\right)=2$. In addition, let $X_{1}$ and $X_{2}$ be a clopen partition of $M_{t} \backslash\{x\}$ and let $Y_{1}$ and $Y_{2}$ be a clopen partition of $M_{t} \backslash\{y\}$ such that $x \in Y_{1}$ and $y \in X_{1}$. Then the interval between $x$ and $y$ will be defined as $I(x, y)=X_{1} \cap Y_{1}$. If $x=y$ then $I(x, y)=\emptyset$.

Remark. By lemma [16, if $V \subset M_{t}$ is an open definable connected subset and $x, y \in V$ then $I(x, y) \cap V \neq \emptyset$ and the following union is disjoint:

$$
V=\left(X_{2} \cap V\right) \cup\{x\} \cup(I(x, y) \cap V) \cup\{y\} \cup\left(Y_{2} \cap V\right)
$$

This motivates us to think of $I(x, y)$ as the set lying "in between" $x$ and $y$.

Lemma 28. Let $M$ be a 1-dimensional connected $\omega$-saturated t.t.t structure such that for every point $x \in M_{t}, d_{M}\left(M_{t} \backslash\{x\}\right)>1$. Let $D \subset M_{t}$ be a connected open definable subset which is locally flat. Let $x \neq y$ be points in D. Then:

(1) $I(x, y) \cap D$ is a non-empty definable open connected set.

(2) $\{x, y\}=b d(I(x, y))$.

(3) If $a, b \in D \backslash\{x, y\}$ such that $a \sim_{x} b$ and $a, b \notin I(x, y)$, then $a \sim_{y} b$.

(4) If $a, b \in D \backslash\{x, y\}$ such that $a \nsim_{x} b$ and $a, b \notin I(x, y)$, then $a \nsim_{y} b$.

Proof. Let $X_{1}$ and $X_{2}$ be the connected components of $M_{t} \backslash\{x\}$ and let $Y_{1}$ and $Y_{2}$ be the connected components of $M_{t} \backslash\{y\}$. Note that by proposition 25, both $D \backslash\{x\}$ and $D \backslash\{y\}$ have exactly two connected components which are given by $X_{1} \cap D$, $X_{2} \cap D$ and $Y_{1} \cap D, Y_{2} \cap D$ respectively. Without loss of generality, $x \in Y_{1}$ and $y \in X_{1}$ so $I(x, y)=X_{1} \cap Y_{1}, X_{2} \subset Y_{1}$ and $Y_{2} \subset X_{1}$. By lemma 16 this means that

(*) $D=\left(X_{2} \cap D\right) \cup\{x\} \cup(I(x, y) \cap D) \cup\{y\} \cup\left(Y_{2} \cap D\right)$.

We'll now prove the four parts of the lemma.

(1) First of all, since $X_{1}$ and $Y_{1}$ are definable, $I(x, y)$ is definable as well.

By lemma 16, $I(x, y) \cap D \neq \emptyset . I(x, y)$ is open as the intersection of open sets. 
Now, assume for contradiction that $I(x, y) \cap D$ isn't connected. Let $A_{1}$ and $A_{2}$ be a clopen partition of $I(x, y) \cap D$. By lemma 16, the boundaries of $A_{1}$ and $A_{2}$ in $D$ are contained in $\{x, y\}$. Since $D$ is connected, for each $i=1,2$ we have either $x \in b d\left(a_{i}\right)$ or $y \in b d\left(A_{i}\right)$. Assume for contradiction that $x \notin \overline{A_{1}}$ and $y \notin \overline{A_{2}}$. By $\left.{ }^{*}\right)$ this means that the sets

$$
\left(D \cap X_{2}\right) \cup\{x\} \cup A_{2},\left(D \cap Y_{2}\right) \cup\{y\} \cup A_{1}
$$

form a clopen partition of $D$ which is a contradiction to the assumption that $D$ is connected.

Therefore, without loss of generality, we can assume that $x \in b d\left(A_{1}\right)$ and $x \in b d\left(A_{2}\right)$. But then the set $U=D \cap Y_{1}$ is open and $U \backslash\{x\}$ has more than two connected components which is a contradiction to lemma 25 .

(2) This follows immediately from lemma 16

(3) If $a, b \in X_{2} \cap D$ then since $X_{2} \subset Y_{1}, a, b \in Y_{1}$ which means that $a \sim_{y} b$. So we can assume that $a, b \in X_{1} \cap D$. By the assumption, $a, b \notin Y_{1}$ which by $\left(^{*}\right)$ implies that $a, b \in Y_{2} \Rightarrow a \sim_{y} b$.

(4) Without loss of generality, $a \in X_{1}$ and $b \in X_{2}$. Therefore, $b \in Y_{1}$. In addition, $Y_{2} \subset X_{1}$ and $a \notin Y_{1} \cap X_{1}$ so $a \in Y_{2}$. This means that $a \nsim_{y} b$.

Lemma 29. Let $M$ be a 1-dimensional connected $\omega$-saturated t.t.t structure such that for all $x \in M_{t}, d_{M}\left(M_{t} \backslash\{x\}\right)>1$. Let $D \subset M_{t}$ be a connected open definable subset which is locally flat. Let $x_{0} \in D$. Then there exist points $a, b \in D$ such that:

(1) $x_{0} \in I(a, b)$

$(2)<_{x_{0}, I(a, b)}$ defines a dense linear order on $I(a, b)$

(3) $I(a, b) \subset D$

(4) For all $x \in I(a, b), a \nsim_{x} b$

Proof. By proposition 26 there exists a definable connected open neighborhood $U \subset D$ of $x_{0}$ such that $<_{x_{0}, U}$ defines a dense linear order on $U$. Since $M_{t}$ is Hausdorff and $b d(U)$ is finite, we can assume that $<_{x_{0}, U}$ defines a dense linear order on $\bar{U}$. Let $a$ be the point on $b d(U)$ such that $x_{0}<_{x_{0}, U} a$ and such that for every point $y \in b d(U)$ with $x_{0}<_{x_{0}, U} y, a \leq_{x_{0}, U} y$. Similarly, Let $b$ be the point on $b d(U)$ such that $b<_{x_{0}, U} x_{0}$ and for each point $y \in b d(U)$ with $y<_{x_{0}, U} x_{0}$, $y \leq_{x_{0}, U} b$.

Let $A_{1}$ and $A_{2}$ be a clopen partition of $M_{t} \backslash\{a\}$ such that $b \in A_{1}$ and let $B_{1}$ and $B_{2}$ be a clopen partition of $M_{t} \backslash\{b\}$ such that $a \in B_{1}$. Furthermore, let $X_{+}$and $X_{-}$be a clopen partition of $M_{t} \backslash\left\{x_{0}\right\}$ such that $a \in X_{+}$and $b \in X_{-}$.

First we prove that $x_{0} \in I(a, b)=A_{1} \cap B_{1}$. Assume for contradiction that $x_{0} \in A_{2}$. Since $a \in X_{+}$it follows from lemma 16 that $X_{-} \subset A_{2}$ which implies that $b \in A_{2}$ which is a contradiction. Similarly, $x_{0} \in B_{1}$. Together this shows that $x_{0} \in A_{1} \cap B_{1}$.

Next we'll prove that for every point $y \in I(a, b) \cap \bar{U}$ we have $a \nsim_{y} b$ and $b<_{x_{0}, U}$ $y<_{x_{0}, U} a$. Let $y$ be some point in $I(a, b) \cap \bar{U}$. Without loss of generality, $y \in X_{+}$. Since in addition $y \in A_{1}, a \nless_{x_{0}, U} y$ which means that $y<_{x_{0}, U} a$. By the definition of the order this implies that $x_{0} \nsim_{y} a$. Let $Y_{1}$ and $Y_{2}$ be a clopen partition of $M_{t} \backslash\{y\}$ such that $x_{0} \in Y_{1}$ and $a \in Y_{2}$. By lemma [16. $X_{-} \subset Y_{1}$ which means that $b \in Y_{1}$. This proves that $y<_{x_{0}, U} a$ and $a \nsim_{y} b$. Similarly, $b<_{x_{0}, U} y$. 
We'll now see that $I(a, b) \subset U$. By lemma 28, $I(x, y) \cap D$ is a non-empty open connected set. Furthermore, as we showed above, $x_{0} \in I(a, b)$. Assume for contradiction that $I(a, b) \backslash U \neq \emptyset$. Then by the connectedness of $I(a, b) \cap D$, $I(a, b) \cap b d(U) \neq \emptyset$. Let $y$ be a point in $I(a, b) \cap b d(U)$. Then as we saw before, $b<_{x_{0}, U} y<_{x_{0}, U} a$ which is clearly a contradiction to the choice of $a$ and $b$.

Lemma 30. Let $M$ be a 1-dimensional connected $\omega$-saturated t.t.t structure such that for every point $x \in M_{t}, d_{M}\left(M_{t} \backslash\{x\}\right)>1$. Let $D \subset M_{t}$ be a connected open definable subset which is locally flat. Let $x_{0}, a$ and $b$ be points in $D$ such that $x_{0} \in I(a, b) \subset D$, for every point $y \in I(a, b)$ we have $a \nsim_{y} b$ and $<_{x_{0}, I(a, b)}$ defines a dense linear order on $I(a, b)$.

Then for each pair of points $c, d \in I(a, b)$ such that $c<_{x_{0}, I(a, b)} d$,

$$
I(c, d)=\left\{y \in I(a, b) \mid c<_{x_{0}, I(a, b)} y<_{x_{0}, I(a, b)} d\right\}
$$

Proof. Let $C_{a}$ and $C_{b}$ be a clopen partition of $M_{t} \backslash\{c\}$ such that $a \in C_{a}$ and $b \in C_{b}$ and let $D_{a}$ and $D_{b}$ be a clopen partition of $M_{t} \backslash\{d\}$ such that $a \in D_{a}$ and $b \in D_{b}$. Furthermore, let $A_{1}$ and $A_{2}$ be a clopen partition of $M_{t} \backslash\{a\}$ such that $c, d, b \in A_{1}$ and let $B_{1}$ and $B_{2}$ be a clopen partition of $M_{t} \backslash\{b\}$ such that $c, d, a \in B_{1}$.

Let $X_{+}$and $X_{-}$be a clopen partition of $M_{t} \backslash\left\{x_{0}\right\}$ such that $a \in X_{-}$and $b \in X_{+}$. We'll assume that $c, d \in X_{+}$since the other cases are either similar or trivial.

Now we'll show that $x_{0} \in C_{a}$ and $x_{0} \in D_{a}$. Assume for contradiction that $x_{0} \in C_{b}$. Since $c \in X_{+}$, it follows from lemma 16 that $X_{-} \cap C_{a}=\emptyset$ which is a contradiction to the fact that $a \in X_{-} \cap C_{a}$. The proof that $x_{0} \in D_{a}$ is similar.

Since $c<_{x_{0}, I(a, b)} d$ and $x_{0} \in C_{a} \cap D_{a}$, by the definition of $<_{x_{0}, I(a, b)}$ it follows that $d \in C_{b}$ and $c \in D_{a}$.

We're now ready to prove the lemma.

First we note that by lemma 16, it follows from the assumptions above that $I(a, b)=A_{1} \cap B_{1}, C_{b} \subset A_{1}$ and $D_{a} \subset B_{1}$. In addition, since $d \in C_{b}$ and $c \in D_{a}$ it follows from lemma 16 that $I(c, d)=C_{b} \cap D_{a}$. Together this means that $I(c, d) \subset$ $I(a, b)$.

We'll now prove that

$$
I(c, d)=\left\{y \in I(a, b) \mid c<_{x_{0}, I(a, b)} y<_{x_{0}, I(a, b)} d\right\}
$$

Let $y$ be some point in $I(a, b)$ such that $c<_{x_{0}, I(a, b)} y<_{x_{0}, I(a, b)} d$. Since $c<_{x_{0}, I(a, b)}$ $y, y \in X_{+}$. In addition, $y \in X_{+} \cap C_{a} \Rightarrow c \nless_{x_{0}, I(a, b)} y$ so $y \in C_{b}$. In a similar fashion it follows that $y \in D_{a}$. Together this means that $y \in I(c, d)$.

Now, let $y$ be a point in $I(c, d)=C_{b} \cap D_{a}$. Since $C_{b} \subset X_{+}, y \in X_{+}$. Furthermore, since $y \in C_{b}, c<_{x_{0}, I(a, b)} y$. Finally, since $y \in D_{a}, d \nless_{x_{0}, I(a, b)} y$ which means that $y<_{x_{0}, I(a, b)} d$.

Now, let's assume that $M$ and the set $D \subset M_{t}$ fulfill the assumptions in lemma 29. Then for each point $x_{0} \in D$ there exists a pair of points $a$ and $b$ in $D$ such that $x_{0} \in I(a, b) \subset D$, for every point $y \in I(a, b)$ we have $a \nsim_{y} b$ and $<_{x_{0}, I(a, b)}$ defines a dense linear order on $I(a, b)$.

By lemma 30, this means that for all $c, d \in I(a, b), I(c, d) \subset I(a, b)$ and:

$$
\left\{y \in I(a, b) \mid c<_{x, V_{x}} y<_{x, V_{x}} d\right\}=I(c, d)
$$

. In other words, in the set $I(a, b)$ guaranteed by lemma 29, the notion of an interval we defined above coincides with the interval induced by the order $<_{x_{0}, I(a, b)}$. 
We'll now prove three lemmas about locally flat points which together will show that the order we defined above can be extended from locally flat points to connected locally flat sets.

Lemma 31. Let $M$ be a 1-dimensional connected $\omega$-saturated t.t.t structure such that for every element $x \in M_{t}, d_{M}\left(M_{t} \backslash\{x\}\right)>1$. Let $D \subset M_{t}$ be a connected open definable subset which is locally flat. Let $x, a$ and $b$ be points in $D$ such that for every open set $U \subset D$ containing $x$ there exists some point $u \in U$ such that $a \nsim_{u} b$. Then $a \nsim_{x} b$.

Proof. Since $M_{t}$ is Hausdorff, there exists an open definable connected set $U \subset$ $D \backslash\{a, b\}$ containing $x$. By lemma 29, there exists a pair of points $c$ and $d$ in $U$ such that $x \in I(c, d) \subset U$, for every point $y \in I(c, d)$ we have $c \nsim_{y} d$ and $<_{x, I(c, d)}$ defines a dense linear order on $I(c, d)$.

By the assumptions of the lemma, there exists some $y \in I(c, d)$ such that $a \nsim_{y} b$. By lemma 30, $I(x, y) \subset I(c, d)$ which means that $a, b \notin I(x, y)$. So by lemma 28, $a \nsim_{x} b$.

Lemma 32. Let $M$ be a 1-dimensional connected $\omega$-saturated t.t.t structure such that for all $x \in M_{t}, d_{M}\left(M_{t} \backslash\{x\}\right)>1$. Let $D \subset M_{t}$ be a connected open definable subset which is locally flat. Let $x, a$ and $b$ be points in $D$ such that $a \nsim_{x} b$. The there exists a definable open set $U \subset D$ containing $x$ such that for every $u \in U$, $a \nsim_{u} b$.

Proof. As in the previous lemma, there exists an open definable connected set $U \subset D \backslash\{a, b\}$ containing $x$. By lemma 29, there exists a pair of points $c$ and $d$ in $U$ such that $x \in I(c, d) \subset U$, for every point $y \in I(c, d)$ we have $c \nsim_{y} d$ and $<_{x, I(c, d)}$ defines a dense linear order on $I(c, d)$.

Let's choose some point $y \in I(c, d)$. Since $a, b \notin I(c, d)$ it follows that $a, b \notin$ $I(x, y) \subset I(c, d)$. Therefore, by lemma 28, $a \nsim_{y} b$.

We now use the previous two lemmas to show that in some well defined sense, locally flat sets look like a line.

Lemma 33. Let $M$ be a 1-dimensional $\omega$-saturated t.t.t structure such that for every point $x \in M_{t}, d_{M}\left(M_{t} \backslash\{x\}\right)>1$. Let $D \subset M_{t}$ be a connected open definable subset which is locally flat. Then, there doesn't exist a definable connected closed subset $F \subset D$ such that bd $(F)>2$.

Proof. Assume for contradiction that $F \subset D$ is a definable closed connected subset and that $a, b, c \in b d(F)$.

Let $F_{a b}$ denote the set of points $x \in F$ such that $a \nsim_{x} b$.

Claim. $F_{a b} \neq \emptyset$.

Proof. By lemma 29, there exists a pair of points $x$ and $y$ in $D$ such that $a \in$ $I(x, y) \subset D$, for every point $z \in I(x, y)$ we have $x \nsim_{z} y$ and $<_{a, I(x, y)}$ defines a dense linear order on $I(x, y)$.

Let $s$ and $t$ be points in $I(x, y)$ such that $s<_{a, I(x, y)} a<_{a, I(x, y)} t$. By lemma 30

$$
\left\{z \in I(a, b) \mid s<_{a, I(x, y)} z<_{a, I(x, y)} t\right\}=I(s, t)
$$

so we can use the notion $I(s, t)$ to represent the interval given by the order $<_{a, I(x, y)}$. We'll use the result throughout the proof of the claim. 
Since $I(s, t)=I(s, a) \cup\{a\} \cup I(a, t)$ and $a \in b d(U)$, we can assume without a loss of generality that for every point $v \in I(a, t)$ there exists a point $u \in I(a, v)$ such that $u \in F$. Therefore, there exists some point $u \in I(a, t)$ such that $I(a, u) \subset F$. Because assume for contradiction that for every $u \in I(a, t)$ there existed some $v \in I(a, u)$ such that $v \notin F$. In that case, the definable set $I(a, t) \cap F$ would have an infinite number of connected components which is a contradiction to $M$ being t.t.t.

Now, since $a \notin \operatorname{int}(F)$, for every point $u \in I(s, a)$ there must be some point $v \in I(u, a)$ such that $v \notin F$. Similarly to above, this means that there exists some point $v \in I(s, a)$ such that $I(v, a) \cap F=\emptyset$.

Together, this means that without loss of generality we can assume that $I(x, a) \cap$ $F=\emptyset$ and $I(a, y) \subset F$.

If $b \in I(a, y)$ then for any $u \in I(a, b) \subset I(a, y) \subset F, a \nsim_{u} b$ and so $u \in F_{a b}$.

Let's assume that $b \notin I(a, y)$.

Let $A_{1}$ and $A_{2}$ be a clopen partition of $M_{t} \backslash\{a\}$ such that $x \in A_{1}$ and let $X_{1}$ and $X_{2}$ be a clopen partition of $M_{t} \backslash\{x\}$ such that $a \in X_{1}$. By the choice of $A_{1}$ and $X_{1}$, $I(x, a)=A_{1} \cap X_{1}$. Furthermore, since $I(x, a) \cap F=\emptyset$ and $U$ is connected, from lemma 16 it follows that $F \subset \overline{A_{2}}$ or $F \subset \overline{X_{2}}$. But $I(a, y) \subset A_{2}$ and $I(a, y) \subset F$ so it must be that $F \subset \overline{A_{2}}=A_{2} \cup\{a\}$. This means that $b \in A_{2}$.

Now, let $u$ be some point in $I(a, y) \subset F$. Let $U_{1}$ and $U_{2}$ be a clopen partition of $M_{t} \backslash\{u\}$ such that $a \in U_{1}$. Assume for contradiction that $b \in U_{1}$. Since $b \in A_{2}$ this means that $b \in A_{2} \cap U_{1}$. But $a \in U_{1}$ and $u \in A_{2}$ which means that $I(a, u)=A_{2} \cap U_{1}$. This implies that $b \in I(a, u) \subset I(a, y)$ which is a contradiction to our assumption. Therefore, $b \in U_{1}$ and $a \in U_{2}$ which means that $u \in F_{a b}$.

This concludes the proof of the claim.

By lemmas 32 and 31, $U_{a b}$ is clopen. Therefore, $U=U_{a b}$.

Similarly, if $U_{a c}$ and $U_{b c}$ are defined in the analogous fashion, $U=U_{a c}=U_{b c}=$ $U_{a b}$. We'll now show that this is a contradiction.

Let's choose a point $x \in \operatorname{int}(U)$. Let $X_{1}$ and $X_{2}$ be the two connected components of $D \backslash\{x\}$. Either $X_{1}$ or $X_{2}$ will contain two out of $a, b$, and $c$. Without loss of generality, $a, b \in X_{1}$. However, since $x \in U_{a b}, a \nsim_{x} b$ which is clearly a contradiction.

We're now ready to show that every locally flat set is o-minimal. In order to do this, we'll extend our previous notion of order from neighborhoods of locally flat points to locally flat sets.

Let $D$ be a definable open connected locally flat set. Let $a \in D$ be some arbitrary point which we'll think of as the center. In addition, let $D_{+}$and $D_{-}$be the two connected components of $D \backslash\{a\}$ which we'll think of as the "positive side" and the "negative side". Finally let $x, y \in D$. We say that $x<_{a, D} y$ if one of the following holds:

$$
\begin{aligned}
& \text { - } x, y \in D_{+} \text {and } a \nsim_{x} y \\
& \text { - } x, y \in D_{-} \text {and } a \nsim_{y} x \\
& \text { - } y \in D_{+} \text {and } x \in D_{-} \\
& \text {- } y=a \text { and } x \in D_{-} \\
& \text {- } x=a \text { and } y \in D_{+}
\end{aligned}
$$

By proposition 21 $<_{a, D}$ is definable. 
The next proposition shows that $<_{a, D}$ defines a dense linear order on $D$ such that the induced interval topology is equivalent to the topology induced by $M_{t}$.

Proposition 34. Let $M$ be a 1-dimensional connected $\omega$-saturated t.t.t structure such that for each point $x \in M_{t}, d_{M}\left(M_{t} \backslash\{x\}\right)>1$. Let $D \subset M_{t}$ be a connected open definable subset which is locally flat and let a be some point in $D$. Then $<_{a, D}$ defines a dense linear order on $D$ such that the induced interval topology is equivalent to the topology induced by $M_{t}$.

Proof. Let $D_{+}, D_{-}$be the sets used in the definition of $<_{a, D}$ above.

Let $x, y, z \in D$. In addition, let $X_{1}$ and $X_{2}$ be the connected components of $D \backslash\{x\}$ such that $a \in X_{1} . Y_{1}, Y_{2}, Z_{1}$ and $Z_{2}$ are defined analogously for $y$ and $z$.

(1) $x \nless_{a, D} y \Rightarrow y<_{a, D} x$ :

We assume that $x, y \in D_{+}$. The other possibilities are either identical or trivial. By the assumption, $y \in X_{1}$. Assume for contradiction that $x \in Y_{1}$. Since $a \in X_{1} \cap Y_{1}$, by lemma 16 both $X_{2}$ and $Y_{2}$ are subsets of $D_{+}$.

Let's define

$$
F=\left(D_{+} \cap X_{1} \cap Y_{1}\right) \cup\{a\} \cup\{x\} \cup\{y\}
$$

. By lemma 16 $F$ is closed and $b d(F) \subset\{a, x, y\}$. We'll now show that $F$ is connected and that $b d(F)=\{a, x, y\}$.

Let $C$ be some connected component of $F$. Since $D$ is connected, $b d(C)=$ $\{a, x, y\}$. For assume for contradiction that one of the points in the set $\{a, x, y\}$ was not included in $b d(C)$. Without loss of generality, let's assume that $b d(C)=\{x, y\}$. Since $b d(C) \subset\{a, x, y\}, b d\left(X_{2}\right)=\{x\}$ and $b d\left(Y_{2}\right)=$ $\{y\}$, it follows that $C \cup X_{2} \cup X_{1} \cup\{x\} \cup\{y\}$ is a clopen subset of $D$ which is clearly a contradiction. Assuming that $b d(C)$ is equal to some other strict subset of $\{a, x, y\}$ gives a similar contradiction.

In addition, $a, x$ and $y$ are boundary points of $D_{-}, X_{2}$ and $Y_{2}$ respectively so by lemma 25] each one of $a, x$, and $y$ is the boundary point of at most one connected component of $F$. Therefore, $F$ has only one connected component and $b d(F)=\{a, x, y\}$.

However, this is a contradiction to lemma 33.

(2) $x<_{a, D} y \Rightarrow y \nless_{a, D} x$ :

Also in this case we'll assume that $x, y \in D_{+}$. Since $x<_{a, D} y$ and $a \in X_{1}$, we get that $y \in X_{2}$. Now, we claim that $x \in Y_{1}$. For otherwise, if it was true that $x \in Y_{2}$ then we'd get from lemma 16 that $Y_{1} \cap X_{1}=\emptyset$ which is a contradiction to the fact that $a \in X_{1} \cap Y_{1}$.

(3) $x<_{a, D} y \wedge y<_{a, D} z \Rightarrow x<_{a, D} z$ :

According to the assumptions, $a, x \in Y_{1}, z \in Y_{2}, a \in X_{1}$ and $y \in X_{2}$. We have to prove that $z \in X_{2}$ as well. But again by lemma 16.

$$
y \in X_{2} \wedge x \in Y_{1} \Rightarrow Y_{2} \subset X_{2} \Rightarrow z \in X_{2}
$$

This shows that $<_{a, D}$ is a linear order. We'll now show that $<_{a, D}$ is dense.

Let's assume that $x<_{a, D} y$. As we showed above, this means that $y \in X_{2}$ and $x \in Y_{1}$. By lemma 28, $X_{2} \cap Y_{1}=I(x, y) \neq \emptyset$. Let $s$ be some point in $I(x, y)$. Since $s \in X_{2} \cap Y_{1}$, it follows from the definition of $<_{a, D}$ that $x<_{a, D} s<_{a, D} y$.

We'll now see that the order topology induced on $D$ by $<_{a, D}$ is equivalent to the topology on $D$ induced by $M_{t}$. 
As a first step, we note that if $x<_{a, D} y$, then

$$
I(x, y) \cap D=\left\{z \in D \mid x<_{a, D} z<_{a, D} y\right\}
$$

. This is immediate from the definitions of $I(x, y)$ and $<_{a, D}$.

Let $U \subset D$ be an open set in $D$ with $x \in U$. By lemma 29, there exists a pair of points $s, t \in U$ such that $x \in I(s, t)$ and $I(s, t) \subset U$. Without loss of generality, $s<{ }_{a, D} t$.

Therefore,

$$
x \in\left\{u \in D \mid s<_{a, D} u<_{a, D} t\right\}=I(s, t) \cap D \subset U
$$

. The other direction is trivial as $I(x, y)$ is open in $D$ for every $x, y \in D$.

We now obtain our primary result as an immediate consequence of propositions 24 and 34 .

Theorem 35. Let $M$ be a 1-dimensional connected $\omega$-saturated t.t.t structure such that for every point $x \in M_{t}, d_{M}\left(M_{t} \backslash\{x\}\right)>1$. Then there exists a finite set $X \subset M_{t}$ such that each of the finite number of connected components of $M_{t} \backslash X$ are o-minimal.

Proof. Let $X$ be the definable set of points in $M_{t}$ which aren't locally flat. By proposition 24, $X$ is finite. Let $D$ be a connected component of $M_{t} \backslash X$. Since there're only a finite number of connected components, $D$ is a connected open definable subset which is locally flat. By proposition 34 there exists a definable dense linear order which induces the topology on $D$. By [1, 6.2], this means that $D$ is o-minimal.

Remark. Note that even if $M_{t}$ isn't connected, we can obtain theorem 35 for any open connected definable subset $D \subset M_{t}$ with the property that removing any point from $D$ splits $D$ into more than one connected component.

Example 36. Let's return to the structure $R_{\text {int }}=\langle\mathbb{R}, I(x, y, z)\rangle$ from example 22 By theorem 35. we should be able to recover the standard order $<$ on $\mathbb{R}$ from $I$.

Let $D=\mathbb{R}$ and let $a$ be some point from $\mathbb{R}$. In addition, let $x$ and $y$ be points in $\mathbb{R}$ such that $a<x<y$. By the construction of, $<_{a, D}$ it's clear that $a<_{a, D} x<_{a, D} y$. By checking the other possibilities for $x$ and $y$ in a similar fashion it's easy to see that $<_{a}, D$ is equivalent to $<$.

\section{Structures Without Splitting}

In this section we look at structures where removing a point doesn't split the structure into more than one connected component. One example of such a structure is the unit circle. Our main goal in this section will be to find alternative topological properties which ensure that the structure is at least locally o-minimal as in the case of the unit circle.

Lemma 37. Let $M$ be an $\omega$-saturated one dimensional t.t.t structure. Let $A$ be a definable open set, $f: A \rightarrow \mathcal{P}\left(M_{t}\right)$ a function such that $f(x)$ is finite for each point $x \in M_{t}$ and $\Gamma$ the graph of $f$. Then for each point $x \in A$, the fiber $(\bar{\Gamma})_{x}$ is finite.

Proof. Let's assume for contradiction that there exists a point $x \in A$ and a sequence of points $\left(y_{i}\right)_{i<\omega}$ in $M_{t}$ such that for every $i<\omega$, every basis set $U \subset A$ containing $x$ and every basis point $V$ containing $y_{i}$, there exists some $z \in U \backslash\{x\}$ such that $f(z) \in V$. 
Claim. For every $i<\omega$ and every basis set $V$ containing $y_{i}$ there exists a basis set $U \subset A$ containing $x$ such that for every basis set $W \subset U$ containing $x$ we have $f(b d(W)) \cap V \neq \emptyset$.

Proof. Let's take some $i<\omega$. Let $V$ be some basis set containing $y_{i}$. Assume for contradiction that for every basis set $U \subset A$ containing $x$ there exists some basis set $W \subset U$ containing $x$ such that $f(b d(W)) \cap V=\emptyset$.

We now define $X=f^{-1}(V) \cap A$. By the definition of $y_{i}$, for every basis set $W$ containing $x, W \cap(X \backslash\{x\}) \neq \emptyset$. Therefore, by the assumption for contradiction there exists a descending sequence of basis sets $\left(W_{i}\right)_{i<\omega}$ such that for all $i<\omega$ :

- $x \in W_{i} \subset X$

- $\overline{W_{i+1}} \subsetneq W_{i}$

- $b d\left(W_{i}\right) \cap X=\emptyset$

Proof. By the last two properties, for every $i<\omega$ the set $W_{i} \backslash W_{i+1}$ is clopen in $X$. But this means that $X$ can be partitioned into an infinite number of definable clopen subsets which is a contradiction.

Now, by the $\omega$-saturation we can assume that there exists some $N<\omega$ such that for every $z \in A,|f(z)|<N$. Similarly, there exists some $B<\omega$ such that for every basis set $V,|b d(V)|<B$. Let $V_{1}, \ldots, V_{N B+1}$ be pairwise disjoint basis sets such that for every $1 \leq i \leq N B+1$ we have $y_{i} \in V_{i}$. By the claim, there exists a basis set $U \subset A$ containing $x$ such that for every basis set $W \subset U$ containing $x$ and every every $1 \leq i \leq N B+1$ we have $f(b d(W)) \cap V_{i} \neq \emptyset$.

Let $W \subset U$ be some basis set. By the definitions of $N$ and $B,|f(b d(W))| \leq N B$ which is a contradiction to the fact that $V_{1}, \ldots, V_{N B+1}$ are pairwise disjoint.

Proposition 38. Let $M$ be an $\omega$-saturated one dimensional t.t.t structure such that there exist a definable continuous function $F: M_{t}^{2} \rightarrow M_{t}$ and a point $a \in M_{t}$ such that for each $x \in M_{t}, F(x, x)=a$ and $F(x, \cdot)$ is injective. Let $f: M_{t} \rightarrow \mathcal{P}\left(M_{t}\right)$ be a function such that for every $x \in M_{t},|f(x)|<\infty$ and $f(x) \neq x$. Let $\Gamma$ be the graph of $f$. Then for every basis set $U \subset M_{t}$, there exists a point $x \in U$ such that $(x, x) \notin \bar{\Gamma}$.

Proof. Assume for contradiction that there exists some basis set $U \subset M_{t}$ such that for every $x \in U,(x, x) \in \bar{\Gamma}$. We now define the function $g: U \rightarrow \mathcal{P}\left(M_{t}\right)$ by

$$
g(x)=\{F(x, y) \mid y \in f(x)\}
$$

In addition, we define the function $h: M_{t} \rightarrow \mathcal{P}(U)$ by

$$
h(y)=g^{-1}(y) \cap U
$$

. Let $\Gamma_{g}$ and $\Gamma_{h}$ be the graphs of $g$ and $h$ respectively.

By our assumption on $F, F(x, x)=a$ for each $x \in U$. Therefore, by the continuity of $F$ together with the assumption that for every $x \in U,(x, x) \in \bar{\Gamma}$, we get that $(x, a) \in \overline{\Gamma_{g}}$ for each $x \in U$.

Furthermore, since for every $x \in U$ we have $f(x) \neq x$ and $F(x, \cdot)$ is injective, $g(x) \neq a$ for all $x \in U$.

We'll now show that there exists an open set $A$ containing $a$ such that for every $y \in A$, the set $h(y)$ is finite. By our assumptions on $f, g(x)$ is finite for every $x \in M_{t}$. Therefore, by the exchange principle there are a finite number of points 
$y \in M_{t}$ such that $h(y)$ is infinite. Furthermore, $h(a)=\emptyset$ so by the Hausdorffness of $M_{t}$, there exists an open set $A$ containing $a$ such that for every $y \in A$, the set $h(y)$ is finite.

In addition, since $(x, a) \in \overline{\Gamma_{g}}$ for each $x \in U$, the fiber $\left(\overline{\Gamma_{h}}\right)_{a}$ is infinite. However, this is a contradiction to lemma 37

Proposition 39. Let $M$ be an $\omega$-saturated one dimensional t.t.t structure such that there exist a definable continuous function $F: M_{t}^{2} \rightarrow M_{t}$ and a point $a \in M_{t}$ such that for each $x \in M_{t}, F(x, x)=a$ and $F(x, \cdot)$ is injective.

Then for every basis set $U$ there exists a basis set $V \subset U$ such that for every point $x \in V$ there exists a basis set $W$ such that $b d(W) \cap V=\{x\}$.

Proof. First of all, without loss of generality we can assume that for every $x \in M_{t}$ there is some basis set $W$ such that $x \in b d(W)$. Because let $X$ be the set of all such points. $X$ is clearly definable. Assume for contradiction that $X^{c}$ has a non empty interior. Let $W$ be a basis set such that $\bar{W} \subset X$. Then $b d(W) \subset X$ which is clearly a contradiction. Therefore, $X^{c}$ is finite.

We define a function $f: U \rightarrow \mathcal{P}\left(M_{t}\right)$ by

$$
f(x)=\{y \neq x \mid \text { there exists a basis set } W \text { such that }\{x, y\} \subset b d(W)\}
$$

Let $N$ be an integer such that for every basis set $U,|b d(U)|<N$. Let $\Gamma$ be the graph of $f$.

We now look at two cases.

For the first case let's assume that there exists a basis set $V \subset U$ such that for each $x \in V,|f(x)|<\infty$. By proposition [38, there exists some basis set $W \subset V$ such that $(W \times W) \cap \Gamma=\emptyset$. This means that for every point $x \in W$, there exists a basis set $A$ such that $b d(A) \cap W=\{x\}$. Because let $x$ be some point in $W$ and let $A$ be a basis set such that $x \in b d(A)$. Since $(W \times W) \cap \Gamma=\emptyset$, the rest of the boundary points of $A$ are not contained in $W$ which means that $b d(A) \cap W=\{x\}$.

For the second case, assume that for every basis set $V \subset U$ there exists some point $x \in V$ such that $f(x)$ is infinite. We now assume for contradiction that there doesn't exist a basis set $V \subset U$ such that for every point $x \in V$, there exists a basis set $W$ such that $b d(W) \cap V=\{x\}$.

In order to get a contradiction, we'll inductively build a sequence of tuples of points, basis sets and functions $\left(x_{i}, V_{i}, f_{i}\right)_{i=1}^{N}$ with the following properties:

- $V_{1}$ is an arbitrary basis set in $U, x_{1}$ is a point in $V_{1}$ such that $f\left(x_{1}\right)$ is infinite and $f_{1}=f$.

- For all $1 \leq i \leq N, f_{i}: U \rightarrow \mathcal{P}\left(M_{t}\right)$ is defined by

$$
\begin{aligned}
f_{i}(x)= & \left\{y \neq x, x_{1}, \ldots, x_{i-1} \mid \text { there exists a basis set } W\right. \\
& \text { such that } \left.\left\{x, x_{1}, \ldots, x_{i-1}, y\right\} \subset b d(W)\right\}
\end{aligned}
$$

- For all $1 \leq i \leq N, x_{i} \in V_{i}$

- For all $1 \leq i \leq N, f_{i}\left(x_{i}\right)$ is infinite.

- For all $i<j, x_{i} \notin V_{j}$

The existence of $\left(x_{1}, V_{1}, f_{1}\right)$ follows immediately from our assumptions in the second case.

Let's assume that we've constructed the sequence up to the $i$-th place. Since $f_{i}\left(x_{i}\right)$ is infinite, there exists some basis set $V_{i+1} \subset f_{i}\left(x_{i}\right)$ such that $x_{i} \notin V_{i+1}$. We define $f_{i+1}$ as above. 
Now, if for all $x \in V_{i+1}$ the set $f_{i+1}(x)$ would be finite then just as in the first case, together with the fact that $i<j \Rightarrow x_{i} \notin V_{j}$, there would exist a basis set $W \subset U$ such that for every $y \in W$, there exists a basis set $A$ such that $b d(A) \cap W=\{y\}$. Therefore, by our assumption for contradiction, there exists some point $x_{i+1} \in$ $V_{i+1}$ such that $f_{i+1}\left(x_{i+1}\right)$ is infinite. Thus we've found a tuple $\left(x_{i+1}, V_{i+1}, f_{i+1}\right)$ satisfying the requirements.

However, the existence of the tuple $\left(x_{N}, V_{N}, f_{N}\right)$ is clearly a contradiction because on the one hand $f_{N}\left(x_{N}\right)$ is infinite but by the definition of $N$, for every point $x \in U$ the set $f_{N}(x)$ is empty.

We'll now prove a similar proposition under the assumption that all of the basis sets have only two points in their boundary.

Lemma 40. Let $X$ be a topological space and let $U \subset X$ and $V \subset X$ be connected open sets such that

$$
b d(U) \cap V=b d(V) \cap U=\emptyset
$$

and $U \neq V$. Then $U \cap V=\emptyset$.

Proof. Let's look at the open set $W=U \cap V$. If $W=\emptyset$ then we're finished.

Let's assume that $W \neq \emptyset$. If $W \neq U$ then since $U$ is connected, the boundary of $W$ in $U$ must be non-empty. Let $x \in U$ be a point in $b d(W)$. Since $x \notin W$, $x \notin V$. But $x \in \bar{W} \subset \bar{V}$ which means that $x \in b d(V)$. This is a contradiction to the fact that $x \in U$. Therefore, $W=U$. Similarly, $W=V$. Together this means that $U=V$ which is a contradiction to the assumption.

Lemma 41. Let $M$ be a t.t.t structure and let $X \subset M_{t}$ be some finite subset. Then there are only a finite number of basis sets $U \subset M_{t}$ such that $b d(U)=X$.

Proof. Let $\mathcal{B}$ be the set of basis sets $U$ such that $b d(U)=X$. Assume for contradiction that $\mathcal{B}$ is infinite. Let $\mathcal{C}$ be defined by

$$
\mathcal{C}=\bigcup_{U \in \mathcal{B}}\left\{C \subset M_{t} \mid C \text { is a connected component of } U\right\}
$$

For each $C \in \mathcal{C}, b d(C) \subset X$. In addition, since $\mathcal{B}$ is infinite, $\mathcal{C}$ is infinite as well. However, by lemma 40, for each pair of connected components $C_{1}, C_{2} \in \mathcal{C}$, $C_{1} \cap C_{2}=\emptyset$. Therefore, the definable set

$$
\bigcup \mathcal{C}=\bigcup \mathcal{B}
$$

can be partitioned into an infinite number of clopen sets which is a contradiction to the fact that $M$ is t.t.t.

Proposition 42. Let $M$ be an $\omega$-saturated one dimensional t.t.t structure such that for every basis set $U,|b d(U)|=2$.

Then for every basis set $U$ there exists a basis set $V \subset U$ such that for every point $x \in V$ there exists a basis set $W$ such that $b d(W) \cap V=\{x\}$.

Proof. As before, without loss of generality we can assume that for every $x \in M_{t}$ there is some basis set $W$ such that $x \in b d(W)$.

We also use the function $f: U \rightarrow \mathcal{P}\left(M_{t}\right)$ defined above by

$$
f(x)=\{y \neq x \mid \text { there exists a basis set } W \text { such that }\{x, y\} \subset b d(W)\}
$$


Let $U$ be some basis set. First let's assume that there exists a point $u \in U$ such that $|f(u) \cap U|=\infty$. Since $M_{t}$ is Hausdorff, there exists some basis set $V \subset f(u) \cap U$ such that $u \notin V . V$ clearly satisfies the requirements of the proposition.

On the other hand, assume that for each point $u \in U,|f(u) \cap U|<\infty$. By lemma 41 this means that there are only a finite number of basis sets $W \subset U$ such that $u \in b d(W)$. By the $\omega$-saturation this means that there exists some number $N \in \mathbb{N}$ such that for each point $u \in U$ there are at most $N$ basis sets $W \subset U$ such that $x \in b d(W)$.

We'll now show using downward induction that there exists a basis set $V \subset U$ such that for every point $v \in V$, there are no basis sets $W \subset V$ such that $v \in b d(W)$ which is clearly a contradiction.

Assume that we found a basis set $V_{i}$ for $0<i \leq N$ such that for every point $v \in V_{i}$, there are at most $i$ basis sets $W \subset U$ such that $x \in b d(W)$. Let $v_{i}$ be some point in $V_{i}$ and let $X$ be the set of points $x \in V_{i}$ such that there exists a basis set $W$ with $v_{i} \in W$ and $x \in b d(W)$. Again by the fact that $M_{t}$ is Hausdorff it follows that $X$ is infinite. We choose $V_{i-1}$ to be some basis set such that $V_{i-1} \subset X$ and $v_{i} \notin V_{i-1}$.

Now, let $x$ be some point in $V_{i-1}$. Since $x \in V_{i}$, there are at most $i$ basis sets $W \subset V_{i}$ such that $x \in b d(W)$. However, one of these sets contains $v_{i}$ which isn't an element in $V_{i-1}$. Therefore, there are at most $i-1$ basis sets $W \subset V_{i-1}$ such that $x \in b d(W)$.

This finishes the downward induction and the proposition.

Theorem 43. Let $M$ be a 1-dimensional $\omega$-saturated t.t.t structure such that one of the following holds:

(1) There exist a definable continuous function $F: M_{t}^{2} \rightarrow M_{t}$ and a point $a \in M_{t}$ such that for each $x \in M_{t}, F(x, x)=a$ and $F(x, \cdot)$ is injective.

(2) For every basis set $U,|b d(U)|=2$.

Then for all but a finite number of points, for every point $x \in M_{t}$ there's a basis set $U$ containing $x$ such that $U$ is o-minimal.

Proof. It's enough to prove that for every basis set $U$ there exists a point $x_{0} \in U$ with an o-minimal neighborhood.

Let $U$ be a basis set. By propositions 39 and 42, there exists a basis set $V \subset U$ such that for every point $x \in V$ there exists a basis set $W$ such that $b d(W) \cap V=$ $\{x\}$. This means that for every point $x \in V, V \backslash\{x\}$ has at least two connected components. Without loss of generality $V$ is connected. By theorem 35 (and the remark immediately after it), this means that after removing a finite number of points from $V$ the remaining connected components are o-minimal. Let $C$ be one of the o-minimal components and let $x_{0}$ be some point in $C$. Clearly $x_{0}$ has an o-minimal neighborhood.

Corollary 44. Let $M$ be an $\omega$-saturated one dimensional t.t.t structure which admits a topological group structure. Then all but a finite number of points have an o-minimal neighborhood.

Proof. We define a function $F: M_{t}^{2} \rightarrow M_{t}$ by

$$
F(x, y)=x-y
$$

The function $F$ clearly satisfies the conditions of theorem 43 . 
Proposition 45. Let $M$ be an $\omega$-saturated one dimensional t.t.t structure such that all but a finite number of points have an o-minimal neighborhood. Let $f: M_{t} \rightarrow M_{t}$ be definable function. Then $f$ is continuous for all but a finite number of points.

Proof. We'll show that in every basis set $U$ there is a point $x_{0} \in U$ such that $f$ is continuous at $x_{0}$.

Let $U$ be a basis set and let $\Gamma$ be the graph of $f$.

If the projection of $\Gamma \cap\left(U \times M_{t}\right)$ onto the second coordinate is finite then there exists a basis set $V \subset U$ such that $f$ is constant on $V$.

On the other hand, if the projection is infinite then there exists some point $y \in M_{t}$ with an o-minimal neighborhood such that for any basis set $V$ containing $y$ there exists some $x \in U$ such that $y \neq f(x) \in V$. Let $V$ be an o-minimal basis set containing $y$. By the choice of $y, f^{-1}(V) \cap U$ is infinite so by the assumption there exists some o-minimal basis set $W \subset U$ such that $f(W) \subset V$. Since both $W$ and $V$ are o-minimal, by the monotonicity theorem there exists some $x_{0} \in W \subset U$ such that $f$ is continuous at $x_{0}$.

We conclude this section by giving two examples of theorem 43 .

Example 46. For the first example we return to the unit circle mentioned in the beginning of the section. We'll look at the structure $S=\left\langle S^{1}, R(x, y, z)\right\rangle$ where $R(x, y, z)$ is true if $x$ and $y$ are not opposite each other and $z$ lies on the short arc between $x$ and $y$. If we define the set of basis sets as

$$
\left\{R^{S}(a, b, z) \mid a, b \in S\right\}
$$

then $S$ is a 1 -dimensional $\omega$-saturated t.t.t structure. In addition, for every basis set $U,|b d(U)|=2$ so by theorem $43 S$ is locally o-minimal. This is indeed true as locally $S$ looks like the structure $R_{\text {int }}$ from example 22 which was shown to be o-minimal.

Example 47. This example is a slight variant of $R_{\text {int }}=\langle\mathbb{R}, I(x, y, z)\rangle$. Let's define the relation $R I(x, y, z)$ in $R=\langle\mathbb{R},+, \cdot, 0,1,<\rangle$ by:

$$
R I(x, y, z) \Longleftrightarrow(x<z<y) \wedge(-1<x-y<1)
$$

So $R I$ is a version of $I$ restricted to intervals with a length of less than 1 .

Let $R \prec \bar{R}$ be an $\omega$-saturated elementary extension and let $\overline{\mathbb{R}}$ be the universe of $\bar{R}$. We define $\bar{R}_{\text {rint }}=\langle\overline{\mathbb{R}}, R I\rangle$ to be the restriction of $\bar{R}$ to the language $\{R I\}$.

Clearly $\bar{R}_{\text {rint }}$ is $\omega$-saturated. In addition, since $\bar{R}$ is o-minimal, $\bar{R}_{\text {rint }}$ is a one dimensional t.t.t structure.

However, for any point $a \in \overline{\mathbb{R}}, \overline{\mathbb{R}} \backslash\{a\}$ has only one definably connected component in $R_{\text {rint }}$. Because assume for contradiction that the sets

$$
\begin{aligned}
& A_{+}=\{x \in \overline{\mathbb{R}} \mid x>a\} \\
& A_{-}=\{x \in \overline{\mathbb{R}} \mid x<a\}
\end{aligned}
$$

were definable in $\bar{R}_{\text {rint }}$ using the constants $c_{1}, \ldots, c_{N}$. Let's define subsets

$$
\begin{aligned}
& \tilde{A}_{+}=\left\{x \in \overline{\mathbb{R}} \mid(x>a) \wedge\left(\forall n<N \forall k\left(x>c_{n}+k\right)\right\}\right. \\
& \tilde{A}_{-}=\left\{x \in \overline{\mathbb{R}} \mid(x<a) \wedge\left(\forall n<N \forall k\left(x<c_{n}+k\right)\right\}\right.
\end{aligned}
$$


By the definition of $R I$, an automorphism of $\overline{\mathbb{R}}$ which swaps $\tilde{A}_{+}$and $\tilde{A}_{-}$is an automorphism of $\bar{R}_{\text {rint }}$ together with the constants $c_{1}, \ldots, c_{N}$ which is a contradiction.

On the other hand, the basis sets of $R_{\text {rint }}$ have two boundary points so by theorem 43, every point in $R_{\text {rint }}$ has an o-minimal neighborhood. This makes sense because for any point $a \in \overline{\mathbb{R}}$ and interval $U$ containing $a$ with a length of less than one, we can define an order on $U$ in the same way that we defined an order on $R_{i n t}$ in example 22

Acknowledgement. I'd like to thank Ehud Hrushovski for providing valuable insights and guidance throughout the time spent working on this paper.

\section{REFERENCES}

1. A. Pillay, First order topological structures and theories, The Journal of Symbolic Logic 52 (1987), no. 3, 763-778.

2. A. Robinson, A note on topological model theory, Fundamenta Mathematicae 81 (1974), 159171.

Department of Mathematics, The Hebrew University of Jerusalem, Jerusalem, 91904, ISRAEL

E-mail address: lowdanie@gmail.com 